\title{
Compound open-channel flows: effects of transverse currents on the flow structure
}

\author{
Sébastien Proust ${ }^{1} \dagger$ and Vladimir I. Nikora ${ }^{2}$ \\ ${ }^{1}$ Irstea, UR RiverLy, Centre de Lyon-Villeurbanne, 5 rue de la Doua CS 20244, 69625 \\ Villeurbanne, France \\ ${ }^{2}$ School of Engineering, University of Aberdeen, Aberdeen AB24 3UE, UK
}

(Received xx; revised $\mathrm{xx}$; accepted $\mathrm{xx}$ )

The structure of free-surface flows in a straight compound channel was investigated in a laboratory flume, consisting of a central smooth-bed main channel (MC) and two adjacent rough-surface floodplains (FPs). The experiments covered both uniform and non-uniform flow conditions, with the latter generated by imposing an imbalance in the discharge distribution between MC and FPs at the flume entrance. The non-uniform cases involved transverse currents directed from MC to FPs and vice-versa. The focus of the study was on assessing the effects of transverse currents on: (i) transverse shear layer and horizontal Kelvin-Helmholtz type coherent structures (KHCSs) forming at the interfaces between MC and FPs; (ii) helical secondary currents (SCs) developing across the channel due to topography-induced flow heterogeneity; and (iii) turbulent large- and very-large-scale motions (VLSMs). Transverse currents can entirely displace the shear layer over FP or in MC, but they do not alter the KHCSs to the same degree, resulting in a mismatch between shear layer extent and KHCSs length-scales. KHCSs emerge once dimensionless velocity shear exceeds a critical value above which KHCSs length-scales increase with the shear. Three well-established SC cells, which are induced by turbulence anisotropy, are observed in uniform flow and non-uniform flow with transverse currents towards FP. They are replaced by a single cell in the presence of a transverse mean flow towards MC. The spectral signatures of VLSMs are visible at the upstream section of the flume but they quickly disappear along the flow being suppressed by simultaneous development of KHCSs and SCs.

Key words: River dynamics, Shear layer turbulence, Shallow water flows

\section{Introduction}

River floods often occur in compound channels, which consist of a main channel and one or two adjacent floodplains (called herein the channel sub-sections). At a border between the main channel (MC) and a floodplain (FP), quasi-two-dimensional coherent structures due to the Kelvin-Helmholtz instability can be often observed (Sellin 1964). These large-scale vortices with a vertical axis are largely responsible for the mass, momentum, and energy exchange between deep and fast flow in the MC and shallower and slower flow over FP, resulting in the FP flow acceleration and MC flow deceleration. The latter can be significant as shown in Sellin (1964, figure 7 ), with a decrease of the maximum velocity in the MC by $25 \%$ compared to a MC flow of same depth but without

$\dagger$ Email address for correspondence: sebastien.proust@irstea.fr 
interaction with the FP flow. The additional flow resistance due to the existence of these vortices was first noted by Zheleznyakov (1965) who called it the 'kinematic effect' of the MC-FP interactions. Since the pioneering works of Sellin (1964) and Zheleznyakov (1965), the structure of uniform flows in straight compound channels has been thoroughly investigated in laboratory flumes (e.g., Nicollet \& Uan 1979; Knight \& Demetriou 1983; Knight \& Shiono 1990; Tominaga \& Nezu 1991; Nezu et al. 1999; Soldini et al. 2004; Ikeda \& McEwan 2009; Stocchino \& Brocchini 2010; Stocchino et al. 2011; Besio et al. 2012; Fernandes et al. 2014; Azevedo et al. 2017; Dupuis et al. 2017a; Truong et al. 2019). In particular, the 'kinematic effect' and the development of the helical secondary currents (SCs) across the channel was found to be strongly dependent on the relative flow depth, $D_{r}$ (ratio of FP flow depth $D_{f}$ to $\mathrm{MC}$ flow depth $D_{m}$ ), and on the relative hydraulic roughness between FP and MC (e.g., $n_{f} / n_{m}$ in terms of Manning's roughness coefficients $n$, where subscripts $f$ and $m$ relate to the FP and MC, respectively).

Owing to the numerous sources of non-uniformity along overflowing rivers, the observed flood flows in compound channels are in fact rarely uniform in the longitudinal direction. Streamwise flow non-uniformity can originate, e.g., from: (i) backwater curve effects (Sturm \& Sadiq 1996; Bousmar 2002); (ii) unbalanced discharge distribution between $\mathrm{MC}$ and $\mathrm{FP}(\mathrm{s})$ at an upstream cross-section of a river reach (e.g., Bousmar et al. 2005; Proust et al. 2013, 2017); (iii) changes in the FP width (e.g., Elliot \& Sellin 1990; Bousmar et al. 2004; Proust 2005; Proust et al. 2006; Das et al. 2019) or in the FP land use (e.g., Dupuis et al. 2017b); (iv) a meandering MC (e.g., Shiono \& Muto 1998); and (v) flow unsteadiness. Flow non-uniformity is typically characterized by longitudinal changes in flow depth and also by transverse currents directed from FP to MC or vice versa. These transverse currents represent a transverse mass exchange quantified by the time- and depth-averaged transverse velocity, $U_{y d}=1 / D \int_{0}^{D} U_{y} d z$, where $U_{y}(z)$ is local mean (i.e., time-averaged) transverse velocity, $D$ is flow depth, $y$ and $z$ are the transverse and vertical (normal to the channel bottom) coordinates, respectively. Note that, under uniform flow conditions, depth-averaged transverse flow in compound channels does not (theoretically) exist (i.e., $U_{y d}=0$ ).

Several important questions arise regarding the presence of the transverse currents in overbank river flows. First, what is the effect of the transverse flow on the shear layer between MC and FP and the horizontal Kelvin-Helmholtz type coherent structures (KHCSs), which are often involved in the bank erosion and lateral transfer of sediments, pollutants and nutrients? Second, what are the conditions for the emergence and development of KHCSs within the shear layer in the presence of flow non-uniformity, bearing in mind that the river conveyance is strongly dependent on the kinematic effect due to KHCSs? Third, what is the effect of the transverse flow on the SCs cells and how this effect depends on the magnitude and direction of the transverse currents? Fourth, does the turbulence structure outside the shear layer exhibit the presence of very large scale motions (VLSMs, Kim \& Adrian (1999)), as observed in non-compound open-channel flows, pipe flows, and boundary layer flows (e.g., Adrian \& Marusic 2012; Cameron et al. 2017)?

The main objective of the present paper is to attempt clarifying these questions. Putting aside the potential effects of non-prismatic geometries, the focus of our experimental study is on a straight compound channel with unchanging roughness parameters in the longitudinal direction. The transverse currents in the experiments are generated by imposing an unbalanced upstream discharge distribution between MC and FPs. This paper complements previous experimental works on non-uniform flows in prismatic and non-prismatic channels (Proust et al. 2013, 2017; Peltier et al. 2013a; Dupuis et al. 2017b), 
(a)

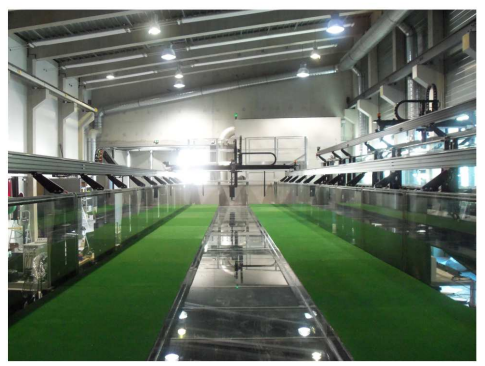

(b)

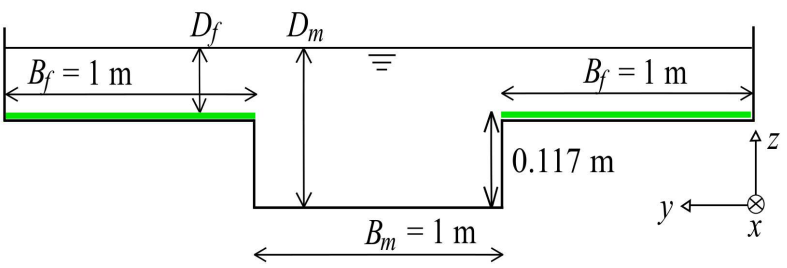

Figure 1. (Colour online) Compound open-channel flume $(18 \mathrm{~m} \times 3 \mathrm{~m})$ at Irstea Lyon-Villeurbanne, France: $(a)$ view upstream; and (b) sketch of a cross-section (view downstream), in which $D_{m}$ and $D_{f}$ are the flow depths in the main channel and floodplain, and $B_{m}$ and $B_{f}$ are the widths of main channel and a floodplain, respectively. Shaded areas (shown in green in online version) represent artificial grass on floodplains.

expanding them in relation to the potential effects associated with the KHCSs and VLSMs. Two specific features of the present work, among others, are worth mentioning at this point: (1) the detection and quantification of the KHCSs using dye tracer and spacetime correlations in both the longitudinal and transverse directions (using two-point velocity measurements); and (2) the assessment of VLSMs presence using long-duration (seven hours) two-point velocity measurements.

Section 2 below outlines the experimental set-up, describes a compound channel flume used in the experiments, flow conditions, and measurement techniques. Section 3 provides information on the streamwise evolution of water depth for all experimental scenarios, as integral characterization of studied flows. The effects of the transverse currents on spanwise shear layer, turbulence statistics, KHCSs, SCs and VLSMs are reported in $\S 4$, 5 , and 6 . The various contributions to the transverse momentum exchange are estimated in $\S 7$, along with their influence on the relaxation towards flow uniformity. Finally, the main conclusions are drawn in $\S 8$.

\section{Experiments}

\subsection{Experimental facility}

The experiments were conducted in an $18 \mathrm{~m}$ long and $3 \mathrm{~m}$ wide compound open-channel flume (figure 1a) at the Hydraulics and Hydro-morphology Laboratory of Irstea, LyonVilleurbanne, France. The flume bed slope in the streamwise direction, $S_{o}$, is $1.1 \times 10^{-3}$. The cross-section consists of a $1 \mathrm{~m}$ wide rectangular glassed-bed MC that is flanked symmetrically by two $1 \mathrm{~m}$ wide flat rough-surface FPs (figures $1 \mathrm{~b}$ and $2 \mathrm{a}$ ), which are covered with dense artificial 'grass' (consisting of $1 \mathrm{~mm}$ wide and $5 \mathrm{~mm}$ high thin rigid blades, with a density of 256 blades per square centimeter). No bending of the grass blades were visually noted in the experiments. Rough-surface FPs were chosen to simulate, to a certain degree, real-life situations, to increase velocity difference between MC and FPs (compared to smooth-bed FPs at the same flow depth) and, subsequently, to enhance planform shear layer turbulence (to be considered in §5). The vertical distance from the MC glass bed to the blades tops on the FP bed is $0.117 \mathrm{~m}$, defining the bank-full stage in the MC. A Cartesian right-handed coordinate system is used in which $x-, y-$, and $z$-axes are aligned with the longitudinal (along the flume), transverse, and vertical (normal to the flume bed) directions (figures $1 \mathrm{~b}$ and $2 \mathrm{~b}$ ). In the following, the longitudinal and lateral distances are normalized by the FP width $\left(x^{*}=x / B_{f}\right.$ and $y^{*}=y / B_{f}$, 
(a)

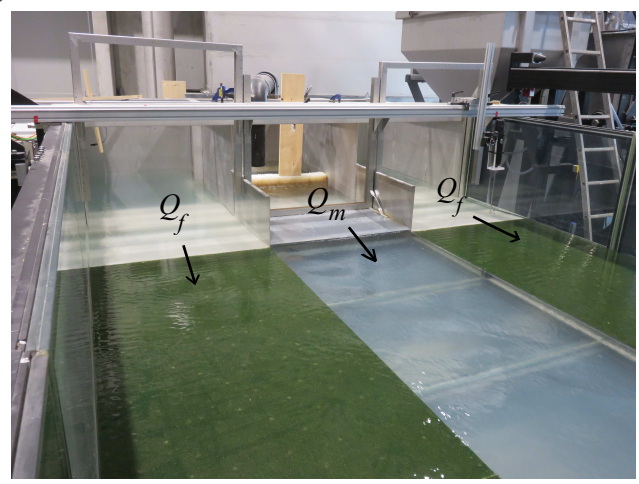

(b)

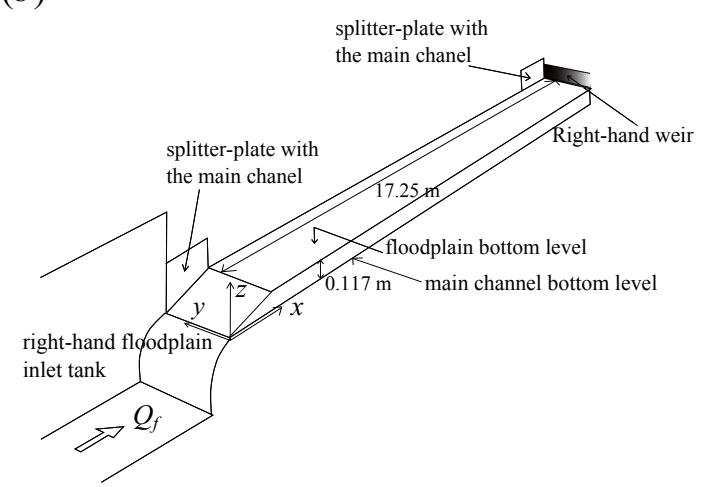

FIGURE 2. (Colour online) Inflow conditions: (a) inlet tanks; $(b)$ sketch of the right-hand floodplain viewed from upstream. The inflow discharge in the main channel is denoted $Q_{m}$, and $Q_{f}$ is the discharge in each of the two floodplains.

figure 1b). The vertical distance is normalized by the MC flow depth under streamwise uniform flow conditions, denoted as $D_{m}^{u}\left(z^{*}=z / D_{m}^{u}\right)$. In the right-handed coordinate system, the origin is defined as (figure $2 \mathrm{~b}$ ): $x^{*}=0$ at the outlet of the three inlet tanks; $y^{*}=0$ at the side-wall of the right-hand $\mathrm{FP}$ (the vertical interfaces between $\mathrm{MC}$ and right-hand and left-hand FPs are thus located at $y^{*}=1$ and $y^{*}=2$, respectively); and $z^{*}=0$ at the MC glass bed.

The inflow set-up is shown in figure 2. The MC, the right-hand and left-hand FPs are supplied with water by three independent inlet tanks (figure 2a), as recommended by Bousmar et al. (2005) based on their experiments. Each inlet tank is $1.7 \mathrm{~m}$ long and 1 $\mathrm{m}$ wide, and is filled with water through a tower with a constant water level reservoir. Each sub-section flow rate $\left(Q_{m}\right.$ in the MC and $Q_{f}$ in each of the two FPs $)$ is monitored with dedicated electromagnetic flow-meters. Within each tank, the flow is accelerated along a transition region with an ellipsoid-shaped bed. At the outlet of each FP inlet tank, a $75 \mathrm{~cm}$ long linear ramp rises the fluid until the FP bed level, as sketched in figure $2 \mathrm{~b}$ for the right-hand FP. Flow partition between MC and FP flows is maintained until $x=0.75 \mathrm{~m}$, i.e., up to the downstream end of the vertical splitter plates (figure $2 \mathrm{~b}$ ).

The effect of the vertical splitter plate on the downstream shear layer development was analyzed in Proust et al. (2017). It was found that the splitter plate induces a long wake with clear velocity deficit in the spanwise profiles of mean velocity if dimensionless velocity shear $\lambda$ (to be considered in $§ 5.4$, Eq. 5.6) is very low, as also observed by Mehta (1991) for free mixing layers (when $\lambda<0.18$ ) or by Constantinescu et al. (2011) for two flows merging at a river confluence with a $\lambda$-value close to 0 . In the present data, the smallest $\lambda$-value $(\leqslant 0.1)$ is observed for the case $20 \mathrm{~L} / \mathrm{s}$ at $x=2.4 \mathrm{~m}(\S 5.4)$. However, even for this extreme case the transverse velocity profiles do not exhibit measurable velocity deficit (to be considered in $\S 4.2$, figure 7 ), and thus the potential effects of the splitter plates can be safely neglected.

At the downstream end of the flume $\left(x^{*}=18\right)$, three variable tail weirs (one per subsection) are used to control the water surface elevation. The adjacent weirs are separated by a $50 \mathrm{~cm}$ long vertical splitter plate (figure $2 \mathrm{~b}$ ).

\subsection{Flow conditions}

The experiments have started with a scenario corresponding to streamwise uniform flow conditions, defined by constant flow depths in the longitudinal direction in each 
sub-section. To achieve such conditions, the inflow discharges $Q_{m}$ and $Q_{f}$ to be injected at $x^{*}=0$ were calculated using the DEBORD formula of Nicollet \& Uan (1979). A uniform flow with a relative flow depth $D_{r}=D_{f}^{u} / D_{m}^{u} \approx 0.2$ was chosen to be studied, as the interaction between the flows in the MC and FPs was found to be the strongest at this $D_{r}$-value (Ackers 1993, p. 115). Given the cross-sectional shape of the flume, its slope, and the Manning roughness coefficients in the sub-sections (estimated in a previous study of Dupuis et al. (2017a)), the flow parameters calculated using the DEBORD formula were: total flow rate $Q=114 \mathrm{~L} \mathrm{~s}^{-1}, D_{r}=0.21, D_{f}^{u}=31 \mathrm{~mm}, D_{m}^{u}=148 \mathrm{~mm}, Q_{m}=$ $98 \mathrm{~L} \mathrm{~s}^{-1}$, and $Q_{f}=8 \mathrm{~L} \mathrm{~s}^{-1}$. The actual (measured) flow parameters achieved via final tuning to uniform flow conditions (table 1, fourth column) appeared to be very close to the predicted values. In the following, each flow case will be identified by its $Q_{f}$-value and thus the uniform flow scenario corresponds to the case of $8 \mathrm{~L} \mathrm{~s}^{-1}$, with $D_{f}^{u}$ varying from $30.6 \mathrm{~mm}$ to $30.5 \mathrm{~mm}$ from $x^{*}=1.2$ to 17.3. This flow case is uniform in terms of flow depth, and features fairly small transverse currents at the MC/FP interfaces in the downstream half of the flume (as shown in section 4.1, figure 5a). On the other hand, it is important to note that in terms of local mean flow velocity the case $8 \mathrm{~L} / \mathrm{s}$ is not uniform, strictly speaking, reflecting streamwise development of the flow structure. The signature of this development can be seen in table 1 that shows the ranges of the time-averaged streamwise velocities outside the shear layer on the low-speed side (i.e., over the FP), $U_{x 1}$, and high-speed side (i.e., in the MC), $U_{x 2}$. As flow case $8 \mathrm{~L} / \mathrm{s}$ does not involve intentionally-induced transverse currents, we consider it as a reference flow termed in this paper 'uniform' or 'depth-uniform'.

Once the measurements for the uniform flow scenario were completed, the experiments continued with non-uniform flows that were generated by imposing an imbalance in the discharge distribution between MC and FPs at the flume entrance, keeping the total flow rate $Q$ the same as for the uniform flow set-up. Five runs with unbalanced inflow conditions have been investigated, with $Q_{f}=0,4,12,16$, and $20 \mathrm{~L} \mathrm{~s}^{-1}$ at each FP, all featuring noticeable changes in the flow depth $D_{f}$ along the flume (table 1 ).

All flow cases are sub-critical in terms of the Froude number and turbulent in terms of the Reynolds number except for $0 \mathrm{~L} / \mathrm{s}$ that is laminar near the flume entrance over the FPs (see Froude numbers $F r_{1}=U_{x 1} / \sqrt{g D_{f}}$ and $F r_{2}=U_{x 2} / \sqrt{g D_{m}}$, and Reynolds numbers $R e_{1}=U_{x 1} D_{f} / \nu$ and $R e_{2}=U_{x 2} D_{m} / \nu$ in table 1$)$. In addition, the Reynolds number $R e_{\delta}$ based on the transverse shear layer width $\delta$ and the half velocity difference $\left(U_{x 2}-U_{x 1}\right) / 2$ was always higher than 2500 . In this range, small-scale three-dimensional (3D) turbulence and quasi-2D KHCSs for plane shear layers co-exist (Lesieur 2013).

\subsection{Water level and velocity measurements}

Water surface elevation was measured using ultrasonic sensors (Baumer UNDK 20I6903/S35A), with a standard measurement error around $0.1 \mathrm{~mm}$. The acquisition duration for each measurement was $200 \mathrm{sec}$ at a rate of $50 \mathrm{~Hz}$. Measurements were taken at spatial intervals of 0.3 to $1 \mathrm{~m}$ in the streamwise direction at transverse positions $y^{*}=0.3$ and 0.7 on the right-hand $\mathrm{FP}$ and at $y^{*}=1.2,1.5$, and 1.8 in the $\mathrm{MC}$ (five streamwise transects in total).

Velocity measurements have been conducted using one-point or two-point acoustic Doppler velocimetry. We have used two 3D Nortek Vectrino+ Acoustic Doppler Velocimeters (ADVs), with side looking probes (sampling volume $5 \mathrm{~cm}$ away from the probe). According to the Nortek specifications, the sampling volume of an ADV can be approximated as a cylinder $6 \mathrm{~mm}$ in diameter and $7 \mathrm{~mm}$ in length. At each measuring point, the three instantaneous velocity components $\left(u_{x}, u_{y}, u_{z}\right)$ were recorded at $100 \mathrm{~Hz}$ 


$\begin{array}{lcccccc}\text { Cases } & 0 \mathrm{~L} \mathrm{~s}^{-1} & 4 \mathrm{~L} \mathrm{~s}^{-1} & 8 \mathrm{~L} \mathrm{~s}^{-1} & 12 \mathrm{~L} \mathrm{~s}^{-1} & 16 \mathrm{~L} \mathrm{~s}^{-1} & 20 \mathrm{~L} \mathrm{~s}^{-1} \\ Q_{f}\left(\mathrm{~L} \mathrm{~s}^{-1}\right) & 0 & 4 & 8 & 12 & 16 & 20 \\ Q_{m}\left(\mathrm{~L} \mathrm{~s}^{-1}\right) & 114 & 106 & 98 & 90 & 82 & 74 \\ \left(Q_{f}-Q_{f}^{u}\right) / Q_{f}^{u}(\%) & -100 \% & -50 \% & 0 \% & +50 \% & +100 \% & +150 \% \\ D_{f}(\mathrm{~mm}) & 17.6-31.3 & 24.2-31.1 & 30.6-30.5 & 34.3-30.9 & 37.7-31.2 & 40.5-31.7 \\ U_{x 1}\left(\mathrm{~cm} \mathrm{~s}^{-1}\right) & 0.00-21.2 & 13.8-22.0 & 25.6-24.7 & 33.7-22.2 & 38.9-24.2 & 44.6-23.8 \\ U_{x 2}\left(\mathrm{~cm} \mathrm{~s}^{-1}\right) & 87.9-84.1 & 74.7-82.9 & 67.2-83.7 & 64.5-81.7 & 58.9-79.5 & 53.4-78.9 \\ F r_{1} & 0.00-0.38 & 0.28-0.40 & 0.46-0.45 & 0.58-0.40 & 0.64-0.44 & 0.72-0.43 \\ F r_{2} & 0.76-0.70 & 0.63-0.69 & 0.56-0.70 & 0.53-0.68 & 0.48-0.66 & 0.43-0.65 \\ \operatorname{Re}_{1} & 0- & 3494- & 7887- & 11399- & 14382- & 17222- \\ & 6614 & 6844 & 7558 & 6844 & 7526 & 7548 \\ \operatorname{Re}_{2} & 119131- & 106345- & 99358- & 97337- & 90767- & 83105- \\ & 124565 & 122717 & 123524 & 120790 & 117657 & 117335 \\ \operatorname{Re}_{\delta} & 14484- & 67551- & 25095- & 18033- & 14492- & 4497- \\ & 139812 & 145857 & 135551 & 110863 & 104625 & 90319\end{array}$

TABLe 1. Flow conditions of the test cases: $Q_{f}$ and $Q_{m}$ are inflows in each of the two FPs and in the MC, respectively, and $Q_{f}^{u}$ is the $Q_{f}$-value for the reference (depth-uniform) case $8 \mathrm{~L} / \mathrm{s}$; ranges of the FP flow depth, $D_{f}$, between $x^{*}=1.2$ and 17.3 ; ranges (between $x^{*}=2.4$ and 16.4) of streamwise time-averaged velocity outside the shear layer on the low-speed side, $U_{x 1}$, and high-speed side, $U_{x 2}$, and associated Froude numbers, $F r_{1}=U_{x 1} / \sqrt{g D_{f}}$ and $F r_{2}=U_{x 2} / \sqrt{g D_{m}}$, and Reynolds numbers, $R e_{1}=U_{x 1} D_{f} / \nu, R e_{2}=U_{x 2} D_{m} / \nu$, and $R e_{\delta}=\left(U_{x 2}-U_{x 1}\right) \delta /(2 \nu)(\nu$ is water kinematic viscosity and $g$ is acceleration due to gravity).

for $300 \mathrm{sec}$ (most measurements) and 7 hours (specifically focused on the identification of long-range velocity fluctuations, as will be explained below). The flow was seeded with polyamide particles (VESTOSINT, manufactured by KVS, Ulm, Germany) with a median diameter of $40 \mu \mathrm{m}$ to increase the signal-to-noise ratio $(\geqslant 22 \mathrm{~dB})$ and the correlation rate within the measuring volume $(\geqslant 90 \%)$. The ADV data were despiked using the phase-space thresholding technique of Goring \& Nikora (2002). The sampling standard errors for the key flow parameters used in this paper were estimated based on 20 time series of $5 \mathrm{~min}$ long each at the same measuring point. These errors are approximately: $1 \%, 9 \%$, and $16 \%$ for the time-averaged velocities, $U_{x}, U_{y}$, and $U_{z}$, respectively; $3 \%, 2 \%$, and $3 \%$ for the turbulence intensities $\sqrt{\overline{u_{x}^{\prime 2}}}, \sqrt{\overline{u_{y}^{\prime 2}}}$, and $\sqrt{\overline{u_{z}^{\prime 2}}}$; and $10 \%$ for the transverse Reynolds shear stress $-\overline{u_{x}^{\prime} u_{y}^{\prime}}$.

One-point velocity measurements were carried out first. Transverse velocity profiles were measured: (a) for $Q_{f}=8 \mathrm{~L} \mathrm{~s}^{-1}$ at elevation $z^{*}=0.94\left(\approx 70 \%\right.$ of $D_{f}^{u}$ from FP bed) and at streamwise positions $x^{*}=2.2,4.2,6.2,8.2,10.2,12.2,14.2,15.8$ and 16.8; and (b) for $Q_{f}=0,4,12,16$, and $20 \mathrm{~L} \mathrm{~s}^{-1}$ at $z^{*}=0.91$ and at $x^{*}=2.4,4.4,8.4,12.4$ and 16.4 (note that extra measurement transects at $x^{*}=6.4$ were added for $16 \mathrm{~L} \mathrm{~s}^{-1}$ and 20 $\mathrm{Ls}^{-1}$ ). In addition, for $Q_{f}=0,4,8,16$ and $20 \mathrm{~L} \mathrm{~s}^{-1}$, full half-cross-sections were covered by velocity measurements at $x^{*}=4.4,8.2$, and 15.9 . Point measurements in the crosssections were taken at intervals of 4 to $10 \mathrm{~mm}$ in the vertical direction (17 $z^{*}$-elevations in the MC, including 5 above the bank-full stage in the MC), and at intervals of 10 to $100 \mathrm{~mm}$ in the lateral direction (16 $y^{*}$-positions in a half-MC, $24 y^{*}$-positions in the right-hand $\mathrm{FP}$ ). Lastly, velocities were measured along the $\mathrm{MC} /$ right-hand $\mathrm{FP}$ interface $\left(\right.$ at $y^{*}=1$ ), at intervals of 4 to $6 \mathrm{~mm}$ along the vertical axis and at $1 \mathrm{~m}$ intervals along 

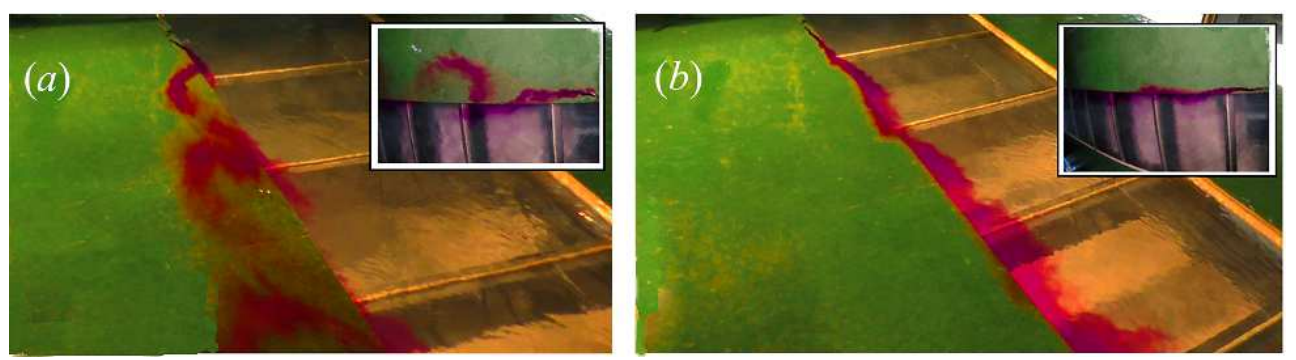

FiguRE 3. (Colour online) Detection of Kelvin-Helmholtz type coherent structures (KHCSs) using a dye tracer that is injected at $x^{*}=6.4$ for the cases of (a) $4 \mathrm{~L} / \mathrm{s}$ and (b) $20 \mathrm{~L} / \mathrm{s}$.

the longitudinal axis, for all flow cases. The ADV measurements very close to the bed were not considered in the analysis, as the ADV probe did not perform well in this region as already observed by Dupuis et al. (2016) in the same flume.

Second, two-point velocity measurements were carried out at elevation $z^{*}=0.91$ for all cases using two ADV probes simultaneously, with two different configurations. In a first step, ADV probes were placed along the transverse direction at a given $x^{*}$-position $\left(x^{*}=2.4,4.4,6.4\right.$ (for $Q_{f}=16$ and $20 \mathrm{~L} / \mathrm{s}$ only), 8.4, 12.4 or 16.4 ). A fixed probe was measuring at the $\mathrm{MC} /$ right-hand $\mathrm{FP}$ interface $\left(y^{*}=1\right)$ while the second probe was moving, point-by-point, along $y^{*}$-axis, across the MC or across the right-hand FP. In a second step, the ADV probes were positioned along the flow at the interface between MC and right-hand $\mathrm{FP}\left(y^{*}=1\right)$. The upstream probe was fixed (measuring at $x^{*}=2.4,4.4$, 6.4 (for $Q_{f}=16$ and $20 \mathrm{~L} / \mathrm{s}$ only), 8.4, 12.4 or 14.9), and the second probe was moving point-by-point downstream. Preliminary measurements have shown that there may be interference between the probes when the transverse distance between them is less than $0.2 \mathrm{~m}$ or when the longitudinal distance between ADVs is less than $0.4 \mathrm{~m}$. The probe separations less than the above distances have been either excluded from the analysis or used for preliminary assessments only.

Finally, to obtain the data for assessing the presence of large (LSMs) and particularly very-large-scale motions (VLSMs), velocity measurements were recorded for $Q_{f}=4$, 8, and $16 \mathrm{~L} / \mathrm{s}$ at $100 \mathrm{~Hz}$ for seven hours at each position. Two ADV probes were simultaneously used, one measuring at the MC center-line $\left(y^{*}=1.5\right)$ at $0.2 D_{m}^{u}$ from the MC bed (elevation at which the VLSMs measured by Cameron et al. (2017) in a non-compound open-channel were found to be sufficiently strong; at the same time any potential effects of KHCSs on VLSMs at this elevation were expected to be minimal), the other measuring in the right-hand FP at $y^{*}=0.35$ and at $0.5 D_{f}^{u}$ from the FP bed. These long-term measurements have been completed at three streamwise positions: $x^{*}=4.4$, 8.4, and 15.9 .

\subsection{Detection of KHCSs using a dye tracer}

To visualize the presence of KHCSs in the flow, we have used a dye tracer (potassium permanganate). As shown in figure 3, this tracer was injected over the FP very near the interface, where large horizontal structures are expected to be generated. Two video cameras were used to get both a global view from the right-hand side of the flume and a top view perpendicular to water surface. 

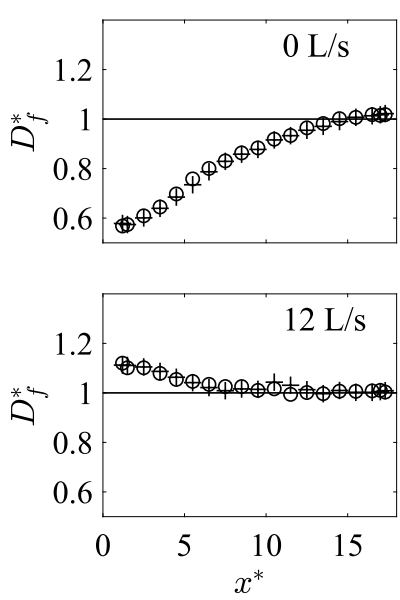
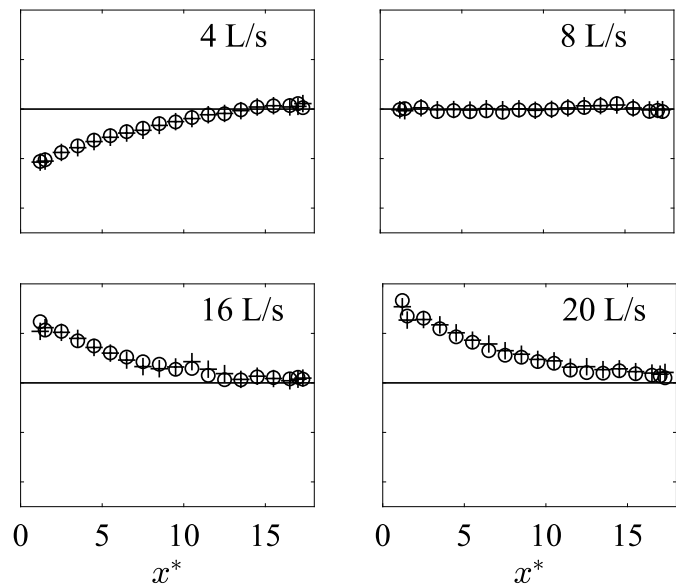

Figure 4 . Dimensionless flow depth, $D_{f}^{*}$, against streamwise coordinate, $x^{*}$, at: $y^{*}=0.3(\circ)$; and $y^{*}=0.7(+)$. The maximum uncertainty in $D_{f}^{*}$ is approximately $8 \times 10^{-3}$.

\section{Streamwise evolution of water depth}

Longitudinal profiles of water depth at two transverse coordinates over the right-hand $\mathrm{FP}$ are plotted in figure 4 . Water depth is normalized as:

$$
D_{f}^{*}=\frac{D_{f}}{<D_{f}^{u}>_{x, y}}
$$

where $D_{f}$ is the local water depth over the FP, and $\left\langle D_{f}^{u}\right\rangle_{x, y}$ is the spatial average of $D_{f}^{u}$ over the streamwise coordinate from $x^{*}=1.2$ to 17.3 and spanwise coordinate from $y^{*}=0.3$ to $y^{*}=0.7$ for the case of $8 \mathrm{~L} \mathrm{~s}^{-1}$.

The case of $8 \mathrm{~L} \mathrm{~s}^{-1}$ features a constant FP flow depth along the whole measuring domain, confirming its streamwise uniformity. The water depth profiles for MC behave in the same way as for FP (not shown here). The five other cases are characterized by significant changes in flow depth in the streamwise direction. The cases with a flow deficit in $\mathrm{FP}$ inflow ( 0 and $4 \mathrm{~L} / \mathrm{s}$ ) exhibit an increase in flow depth along the flow, while the cases with an excess in FP inflow $(12,16$ and $20 \mathrm{~L} / \mathrm{s})$ demonstrate a flow depth decrease. Let us assume that the uniform flow depth (case $8 \mathrm{~L} / \mathrm{s}$ ) is reached when $\left|D_{f}^{*}-1\right| \leqslant 0.010$ (see Eq. 3.1). With this definition, figure 4 shows that the uniform flow depth is reached within the measuring domain for 0,4 , and $12 \mathrm{~L} / \mathrm{s}$. However, no complete uniformity in flow depth is observed for 16 and $20 \mathrm{~L} / \mathrm{s}$. Similar results were obtained for the MC (not shown here). These trends reveal asymmetry in the relaxation towards flow uniformity depending on the direction of transverse currents, e.g., the uniform flow depth is reached over a shorter distance for $0 \mathrm{~L} / \mathrm{s}$ compared to $16 \mathrm{~L} / \mathrm{s}$, although the same amount of water has to be transferred from either side of the interface. The causes of this asymmetry will be analyzed in section 7 .

Figure 4 also shows that for given compound geometry and downstream boundary condition, the flow depth and conveyance at a particular streamwise position are significantly affected by the degree of flow non-uniformity, i.e., by the discharge distribution between $\mathrm{MC}$ and FP. In the upstream part of the flume, the relative difference between the 'non-uniform' flow depth and the uniform flow depth ranges from $-30 \%$ to $+40 \%$. 
(a)

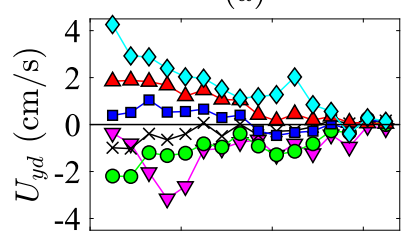

$(d)$

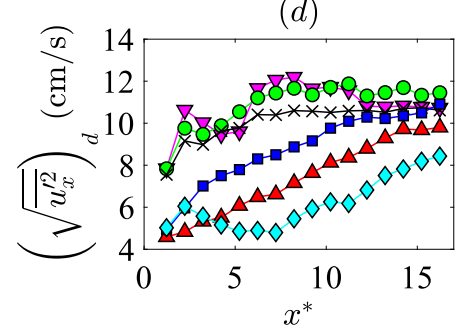

(b)

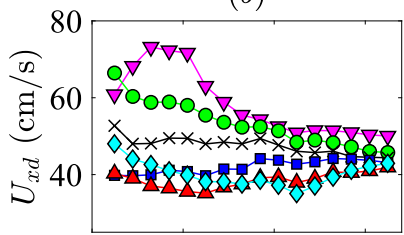

(e)

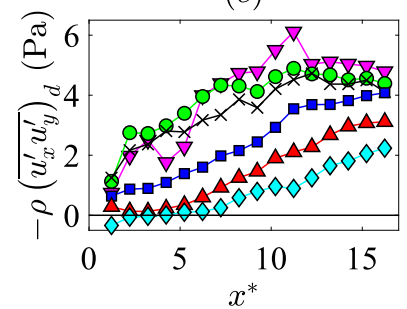

(c)

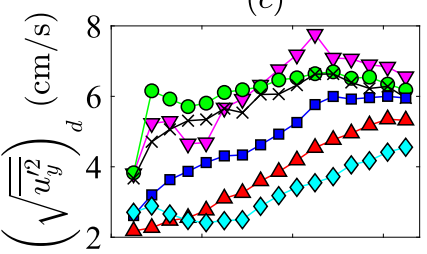

$(f)$

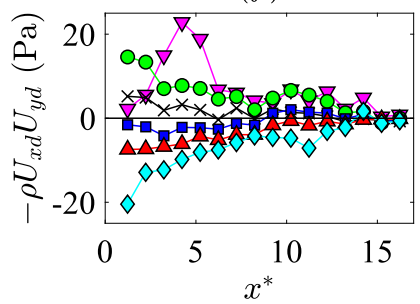

FiguRE 5. (Colour online). Depth-averaged (a) transverse and (b) streamwise mean velocities, depth-averaged $(c)$ transverse and $(d)$ streamwise turbulence intensities, $(e)$ depth-averaged transverse Reynolds shear stress, and $(f)$ transverse flux of streamwise momentum by the depth-averaged flow. Measurements are along the MC/Right-FP interface for cases: $0 \mathrm{~L} / \mathrm{s}(\nabla)$; $4 \mathrm{~L} / \mathrm{s}(\circ) ; 8 \mathrm{~L} / \mathrm{s}(\times) ; 12 \mathrm{~L} / \mathrm{s}(\square) ; 16 \mathrm{~L} / \mathrm{s}(\triangle)$; and $20 \mathrm{~L} / \mathrm{s}(\diamond)$. The standard sampling errors in $U_{x}, U_{y}, \sqrt{\overline{u_{x}^{\prime 2}}}, \sqrt{\overline{u_{y}^{\prime 2}}}$, and $-\overline{u_{x}^{\prime} u_{y}^{\prime}}$ are around $1 \%, 9 \%, 3 \%, 2 \%$, and $10 \%$, respectively.

The channel conveyance is higher in the case of a deficit in FP flow than of an excess in FP flow, highlighting that energy dissipation across the compound section is higher in the latter case than in the former.

\section{Time-averaged flow and turbulence statistics}

\subsection{Depth-averaged velocity at the interface}

Figures $5 \mathrm{a}$ and $5 \mathrm{~b}$ respectively show the depth-averaged transverse and streamwise velocities along the $\mathrm{MC} /$ right-FP interface. The case of $8 \mathrm{~L} / \mathrm{s}$, which is uniform in terms of flow depth (figure 4), exhibits change in the depth-averaged streamwise velocity $U_{x d}$ along the whole measuring domain (figure 5b), reflecting the continuing development of the shear layer (§4.3). This development is accompanied with small negative values of transverse velocity $U_{y d}$, mostly along the upstream half of the flume (figure 5a). These negative $U_{y d}$-values correspond to a weak mass transfer from MC to FP, which may be caused by: (a) a small underestimation of the FP inflow required for equilibrium conditions with zero transverse mass exchange between MC and FP; or/and (b) the uniform distribution across the channel of the streamwise mean velocity at the outlet of each of the three inlet tanks (at $x^{*}=0$ ).

Introducing unbalanced partitioning of the water discharge in the inlet tanks leads to the emergence of transverse currents which magnitude grows with increasing imbalance and which spatial extent reaches the flume length (figure 5a). For the two extreme cases $(0$ and $20 \mathrm{~L} / \mathrm{s})$, the $U_{y d}$-values attain $\approx 5 \%$ and $\approx 9 \%$ of the $U_{x d}$-values, respectively.

\subsection{Transverse profiles of time-averaged streamwise velocity}

Transverse profiles of local time-averaged streamwise velocity, $U_{x}$, for the reference (depth-uniform) flow are shown in figure 6a. Measurements were taken at a fixed elevation $z^{*}=0.94$, i.e., at $\approx 70 \%$ of the FP flow depth $D_{f}^{u}$ from the FP bed. Similarly to Stocchino 

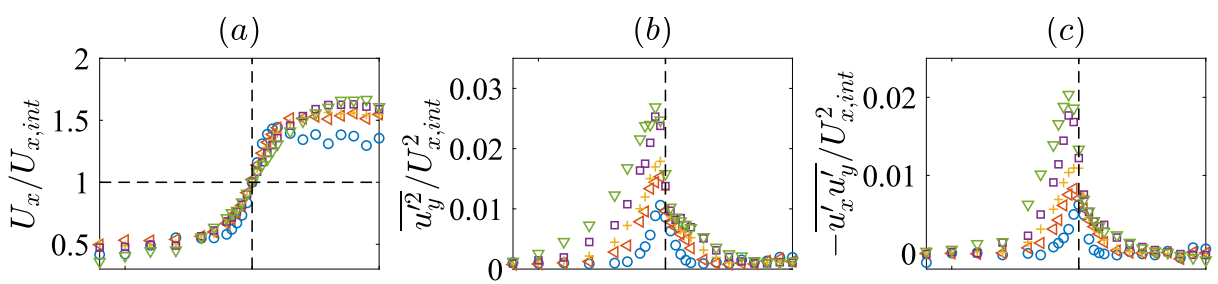

$x^{*}=$

$\circ 2.2$

$\triangleleft 4.2$

$+6.2$

- 8.2
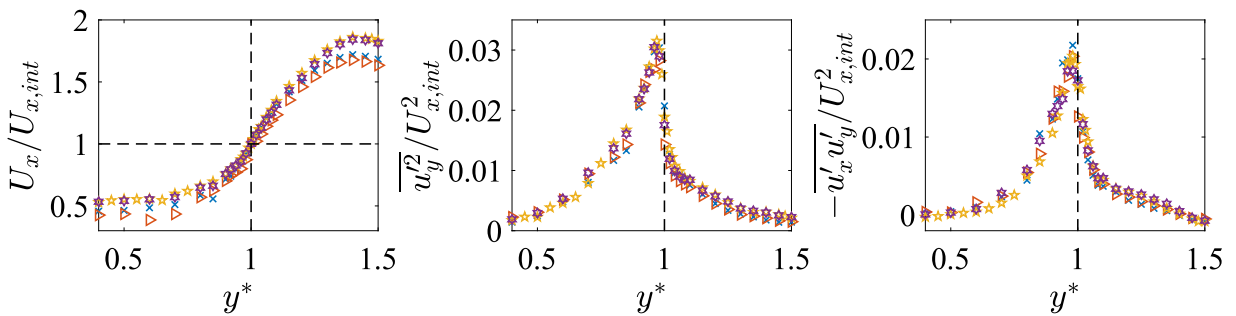

$\nabla 10.2$

$\times 12.2$

$\triangleright 14.2$

* 15.8

* 16.8

FiguRE 6. (Colour online) Transverse distributions of dimensionless (a) mean streamwise velocity, $U_{x} / U_{x, \text { int }}$, (b) transverse squared turbulence intensity, $\overline{u_{y}^{\prime 2}} / U_{x, \text { int }}^{2}$, and (c) transverse Reynolds shear stress, $-\overline{u_{x}^{\prime} u_{y}^{\prime}} / U_{x, i n t}^{2}$, all at elevation $z^{*}=0.94$ for the case of $8 \mathrm{~L} \mathrm{~s}^{-1}$. The standard errors in $U_{x}, \overline{u_{y}^{\prime 2}}$, and $-\overline{u_{x}^{\prime} u_{y}^{\prime}}$ are around $1 \%, 4 \%$, and $10 \%$, respectively.

\& Brocchini (2010) and Dupuis et al. (2017b), the velocity scale used to normalize local time-averaged velocities (and turbulence quantities in the sequel) is the time-averaged streamwise velocity at the MC/FP interface, $U_{x, \text { int }}$. Note that: (1) Dupuis et al. (2017a) found the depth-averaged value of $U_{x, \text { int }}$ to be very close to the convection velocity of the KHCSs that may populate the interfacial region; and (2) the velocity scale $U_{x 2}-U_{x 1}$ used to normalize velocity data for free mixing layers is not sufficiently robust for our case as it attains very small values in some of our experiments (e.g., 16 and $20 \mathrm{~L} / \mathrm{s}$ ) and therefore the interface velocity is more appropriate for normalizations.

The time-averaged velocity profiles are monotonic with an inflection point near the interface where $y^{*}=1$. According to Nezu et al. (1999) and Stocchino \& Brocchini (2010) who classified time-averaged velocity profiles for uniform flows depending on the $D_{r}$-value, the case of $8 \mathrm{~L} \mathrm{~s}^{-1}$ belongs to the 'shallow flow regime', i.e., when $D_{r} \leqslant 0.37$ in Nezu et al. (1999) or $D_{r} \leqslant 0.33$ in Stocchino \& Brocchini (2010) while in our case $D_{r}=0.21$. The monotonic velocity profiles at this flow regime are associated with largescale vortical structures in the horizontal plane that rotate clockwise in the right-hand interfacial region (Stocchino \& Brocchini 2010). The location of the inflection point at $y^{*} \approx 1$, where the large-scale structures are generated, highlights the role played by a sudden change in topography on the generation of these structures (e.g., Soldini et al. 2004).

In the presence of transverse currents, the cross-flow distribution of streamwise mean velocity can be strongly modified as shown in figure 7 for the cases of 0 and $20 \mathrm{~L} / \mathrm{s}$. In particular, the shear layer, defined using mean velocity distributions, is displaced in the direction of the transverse currents. For instance, with transverse currents towards MC $(20 \mathrm{~L} / \mathrm{s})$, the shear layer is nearly entirely shifted in the MC (figure $7 \mathrm{~d}$ ). This interplay between transverse currents and shear layer will be further described in the next section.

\subsection{Shear layer width}

To illustrate the longitudinal evolution of the streamwise time-averaged flow, half the shear layer width, $\delta_{0} / 2$, at a given $z^{*}$-elevation, is shown in figure 8 . To quantify the transverse size of the shear layer we use the definition of van Prooijen et al. (2005): 
(a)

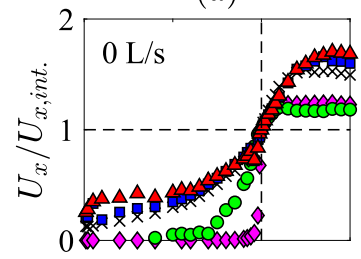

(d)

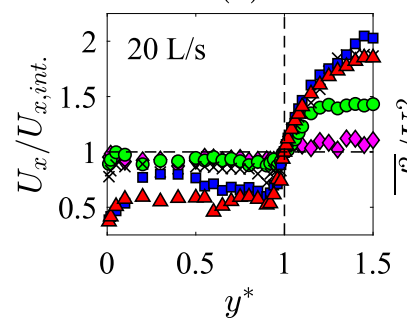

(b)

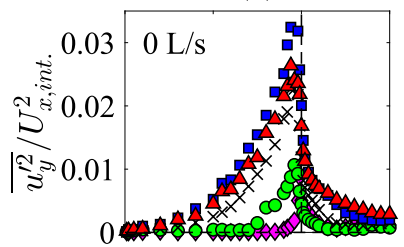

$(e)$

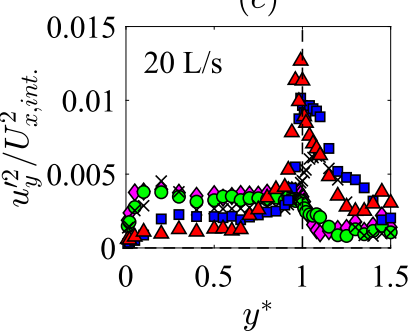

(c)

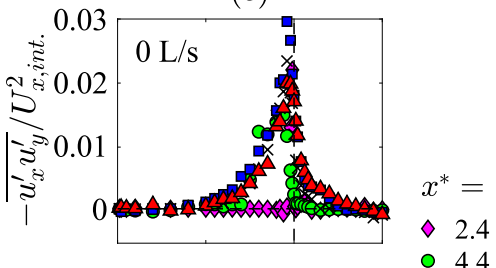

○ 4.4

$\times 8.4$

- 12.4

$\triangle 16.4$

FiguRE 7. Transverse profiles of dimensionless time-averaged streamwise velocity, $U_{x} / U_{x, \text { int }}$, transverse squared turbulence intensity, $\overline{u_{y}^{\prime 2}} / U_{x, i n t}^{2}$ and transverse Reynolds shear stress, $-\left(\overline{u_{x}^{\prime} u_{y}^{\prime}}\right) / U_{x, \text { int }}^{2}$, at various $x^{*}$-positions and at $z^{*}=0.91$ for the cases of (a-c) $0 \mathrm{~L} / \mathrm{s}$, and (d-f) $20 \mathrm{~L} / \mathrm{s}$. The standard errors in $U_{x}, \overline{u_{y}^{\prime 2}}$, and $-\overline{u_{x}^{\prime} u_{y}^{\prime}}$ are around $1 \%, 4 \%$, and $10 \%$, respectively.

$$
\delta_{0}=2\left(y_{75 \%}-y_{25 \%}\right)
$$

where $y_{25 \%}$ corresponds to a location where $U_{x}\left(y_{25 \%}\right)=U_{x 1}+0.25\left(U_{x 2}-U_{x 1}\right)$ and $y_{75 \%}$ is a location where $U_{x}\left(y_{75 \%}\right)=U_{x 1}+0.75\left(U_{x 2}-U_{x 1}\right)$. Here, $U_{x 1}$ is the streamwise velocity averaged across the plateau region of $U_{x}=f\left(y^{*}\right)$ over the right-hand $\mathrm{FP}$, and $U_{x 2}$ is the peak streamwise velocity in the MC.

The data for the reference case of $8 \mathrm{~L} \mathrm{~s}^{-1}$ (figures 6,8 ) highlight three important differences from the unbounded plane free shear layer (see e.g., Champagne et al. 1976; Oster \& Wygnanski 1982): (i) the downstream linear growth of the shear layer is observed only in the MC while its transverse development over the FP is saturated at mid-length of the flume; (ii) the shear layer expands more rapidly on the high velocity side of the shear region compared to the low velocity side; and (iii) the position $y_{50 \%}$ of the mean streamwise velocity $U_{x}\left(y_{50 \%}\right)=U_{x 1}+0.5\left(U_{x 2}-U_{x 1}\right)$ shifts away from the position of the inflection point $\left(y^{*} \approx 1\right)$ into $\mathrm{MC}$ when moving downstream.

It should be noted that the asymmetry of the shear layer for uniform flows in compound open-channels was recently highlighted and analyzed by Dupuis et al. (2017a). To interpret the differences (i) and (ii), we may recall the works of Chu \& Babarutsi (1988) and Uijttewaal \& Booij (2000) on shallow mixing layers in non-compound open-channels and assume that the transverse development of shear layer (horizontal) turbulence is constrained by the strong vertical flow confinement over the FP and that its expansion is stronger suppressed by the vertical bed-induced turbulence over the FP than in the $\mathrm{MC}$ at a given $z^{*}$-elevation. However, the analysis of the KHCSs is required to confirm or reject this hypothesis (as discussed in $\S 5$ ).

In the presence of transverse currents, the data in figure 8 indicate that the shear layer is displaced in the direction of the transverse currents (as shown by arrows), with the case of $8 \mathrm{~L} / \mathrm{s}$ given as the reference. The effects of transverse currents on the shear layer 

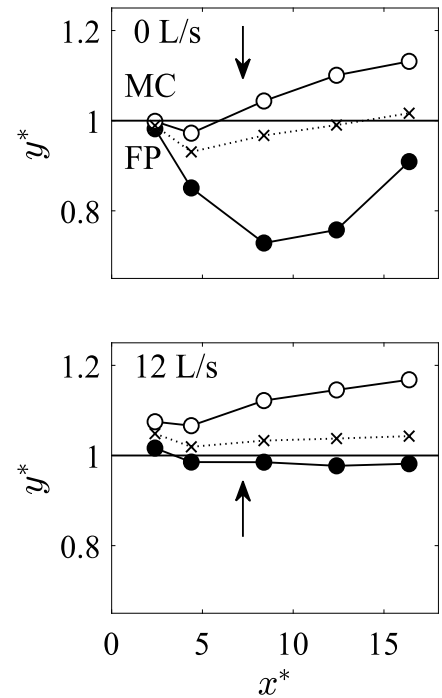
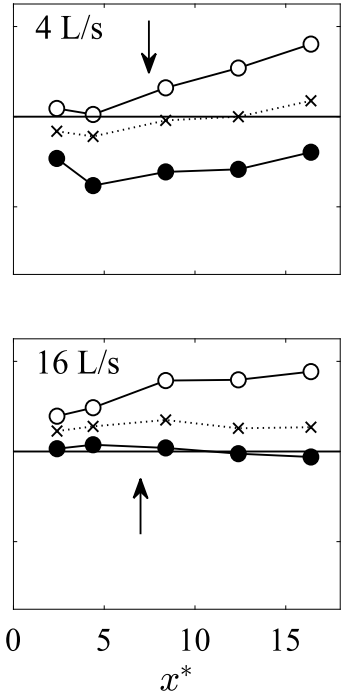
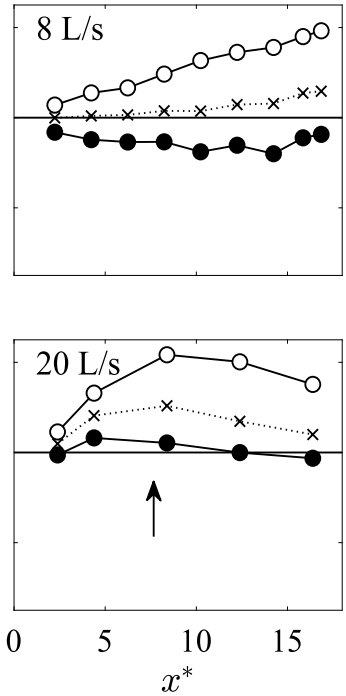

FiguRE 8 . Half shear layer width, $\delta_{0} / 2$ (normalized by $B_{f}$ ), bounded by the transverse positions: $y_{25 \%}(\bullet)$; and $y_{75 \%}(\circ)$. Position $y_{50 \%}(\times)$ is also plotted. Measurements are at $z^{*}=0.94$ for the case of $8 \mathrm{~L} / \mathrm{s}$, and at $z^{*}=0.91$ for the other cases. Arrows indicate the direction of transverse currents.

are particularly noticeable at small $x^{*}$, e.g., at $x^{*}=4.4$ the three-quarters of the shear layer for the case of $4 \mathrm{~L} / \mathrm{s}$ are located over the $\mathrm{FP}$ while for the case of $16 \mathrm{~L} / \mathrm{s}$ the threequarters of the shear layer are sited in the MC. This significant lateral displacement of the shear layer is caused by the high cross-flow momentum exchange via the transverse currents (as reflected in high values of $-\rho U_{x d} U_{y d}$ in figure $5 \mathrm{f}$ ). For the extreme cases of 0 and $20 \mathrm{~L} / \mathrm{s}$ (figure $5 \mathrm{a}$ ), values of $-\rho U_{x d} U_{y d}$ are even one order of magnitude higher than the depth-averaged Reynolds shear stresses (figure 5e).

Further downstream, the effects of transverse currents directed to the MC $(12,16$ and $20 \mathrm{~L} / \mathrm{s}$ ) can still be seen along the whole measurement domain, as the shear layers remain mostly within the MC. With the transverse currents towards the FPs $(0$ and $4 \mathrm{~L} / \mathrm{s})$, the recovery of the shear layer over the $\mathrm{FP}$ to the depth-uniform case $8 \mathrm{~L} / \mathrm{s}$ appears to be faster compared to the opposite direction of the transverse currents $(12,16,20 \mathrm{~L} / \mathrm{s})$, as observed at the downstream part of the flume (figure 8). The similar asymmetry in the relaxation towards uniformity (depending on the direction of transverse currents) is already noted in $\S 3$ when considering streamwise profiles of the flow depth. The causes of this asymmetry will be analyzed in $\S 7$.

\subsection{Turbulence statistics}

For the depth-uniform reference case $(8 \mathrm{~L} / \mathrm{s})$, the spanwise profiles of normalized turbulence statistics are shown in figure 6 . The transverse turbulence intensity $\overline{u_{y}^{\prime 2}} / U_{x, \text { int }}^{2}$ and Reynolds stress $-\overline{u_{x}^{\prime} u_{y}^{\prime}} / U_{x, i n t}^{2}$ rapidly evolve from $x^{*}=2.2$ to 10.2 ; then from $x^{*}=$ 12.2 to 16.8 only a very weak increase in $\overline{u_{y}^{\prime 2}} / U_{x, \text { int }}^{2}$ and $-\overline{u_{x}^{\prime} u_{y}^{\prime}} / U_{x, \text { int }}^{2}$ can be observed, mostly in the MC. This result is consistent with the streamwise evolution of the KHCSs along the flume, as it will be demonstrated in $\S 5$.

In the presence of transverse currents, the cross-flow profiles of normalized turbulence statistics are shown in figure 7 for cases 0 and $20 \mathrm{~L} / \mathrm{s}$. For both flow cases, at a given $x^{*}$-position, the lateral extent of the region of high turbulence intensities and 
Reynolds shear stresses matches well the shear layer width, defined in the previous section based on the mean velocity profiles. In particular, at the $x^{*}$-positions where transverse currents are significant (figure 5a), the same transverse displacement is observed for $-\left(\overline{u_{x}^{\prime} u_{y}^{\prime}}\right) / U_{x, i n t}^{2}, \overline{u_{y}^{\prime 2}} / U_{x, \text { int }}^{2}$ and the streamwise mean velocity profiles. For instance, for $0 \mathrm{~L} / \mathrm{s}$ at $x^{*}=4.4$, the turbulence statistics (figures $7 \mathrm{~b}-\mathrm{c}$ ) are mostly located over the $\mathrm{FP}$, similar to the shear layer (figures 8 and $7 \mathrm{a}$ ) while for $20 \mathrm{~L} / \mathrm{s}$, the high values of $-\left(\overline{u_{x}^{\prime} u_{y}^{\prime}}\right) / U_{x, \text { int }}^{2}$ and $\overline{u_{y}^{\prime 2}} / U_{x, \text { int }}^{2}$ remain sited in the MC all along the measuring domain (figures $7 \mathrm{e}-\mathrm{f}$ ), as observed for the shear layer defined using mean velocities (figures 8 and $7 \mathrm{~d})$.

Another important feature to note is that at 16 and $20 \mathrm{~L} / \mathrm{s}$ the transverse Reynolds stresses at the MC-FP interface are very low within first five meters from the flume entrance (figure 5e), even though the difference $U_{x 2}-U_{x 1}$ is not zero (table 1 ). This difference from other cases is due to the absence of KHCS in this flow section, as it will be shown in $\S 5$ (figure 15).

\subsection{Secondary currents}

It should be noted from the start that the time-averaged transverse velocity, $U_{y}$, and vertical velocity, $U_{z}$, are small compared to the streamwise velocity and thus a potential misalignment of the ADV probe can have a strong impact on the measured values of $U_{y}$ and $U_{z}$ (e.g., Peltier et al. 2013b). The data of $U_{y}$ and $U_{z}$ were therefore corrected. For the spanwise component, we assumed that the cross-sectional average of $U_{y}$-values in the half-MC equals to zero for the depth-uniform flow case $(8 \mathrm{~L} / \mathrm{s})$ at $x^{*}=15.9$. This procedure resulted in a rotation around the vertical axis of a yaw angle $\theta_{z}=0.8^{\circ}$. The same procedure was not applied to the $U_{z}$-component, as this velocity could not be measured within $1 \mathrm{~cm}$ thick near-surface layer. For this component, we assumed that $U_{z}$ should tend towards zero close to the channel bed. Employment of this condition led to slight rotations around the lateral axis (e.g., a pitch angle $\theta_{y} \approx 2^{\circ}$ for cases 20 and $4 \mathrm{~L} / \mathrm{s}$ at $\left.x^{*}=4.4\right)$.

For case $8 \mathrm{~L} / \mathrm{s}$, the distributions of $U_{y}$ and $U_{z}$ in $\mathrm{MC}$ at various $y^{*}$-coordinates at $x^{*}=15.9$ (figure 9a) suggest the existence of two SCs cells: a large cell caused by the anisotropy of the planform shear layer turbulence, and a smaller cell near the bottom at the MC corner induced by topography. The negative values of $U_{z}$ over the water column from $y^{*}=1.3$ to 1.5 indicate that the large cell extends over the entire flow depth in this region, as sketched in figure 9c.

For the same flow, two transverse profiles of $U_{y}$ (at elevation $z^{*}=0.94$ ) are shown in figure $9 \mathrm{~b}$ : data at $x^{*}=4.2$; and longitudinally averaged data $\left\langle U_{y}\right\rangle_{x}$ (average between $x^{*}=4.2$ and 16.8). First, the data in MC reveal that the development of the large SC cell is not fully established at $x^{*}=4.2$. Second, figure $9 \mathrm{~b}$ indicates the existence of a persistent SC cell near the interface on the FP side, termed the 'longitudinal FP vortex' by Tominaga \& Nezu (1991). The $U_{y}$-distribution at $x^{*}=4.2$ within FP is very close to the streamwise-averaged profile indicating that this vortex is already fully developed in the upstream part of the flow. Tominaga \& Nezu (1991) observed a similar vortical motion for a relative depth $D_{r}=0.5$ and for both rough and smooth FPs, for $D_{r}=0.75$ with smooth FPs, but not for $D_{r}=0.25$ and smooth FPs. They therefore concluded that SCs were more driven by the cross-sectional topography (i.e., by $D_{r}$ ) rather than by wall roughness. The present data with $D_{r}=0.2$ and rough FPs, which reveal a strong longitudinal vortex with ratio $U_{y} / U_{x}$ reaching $5 \%$, suggest that wall roughness stimulates the emergence of the FP longitudinal vortex even when $D_{r}$ becomes small.

In the presence of transverse currents towards MC, the SCs pattern radically differs 
(a)
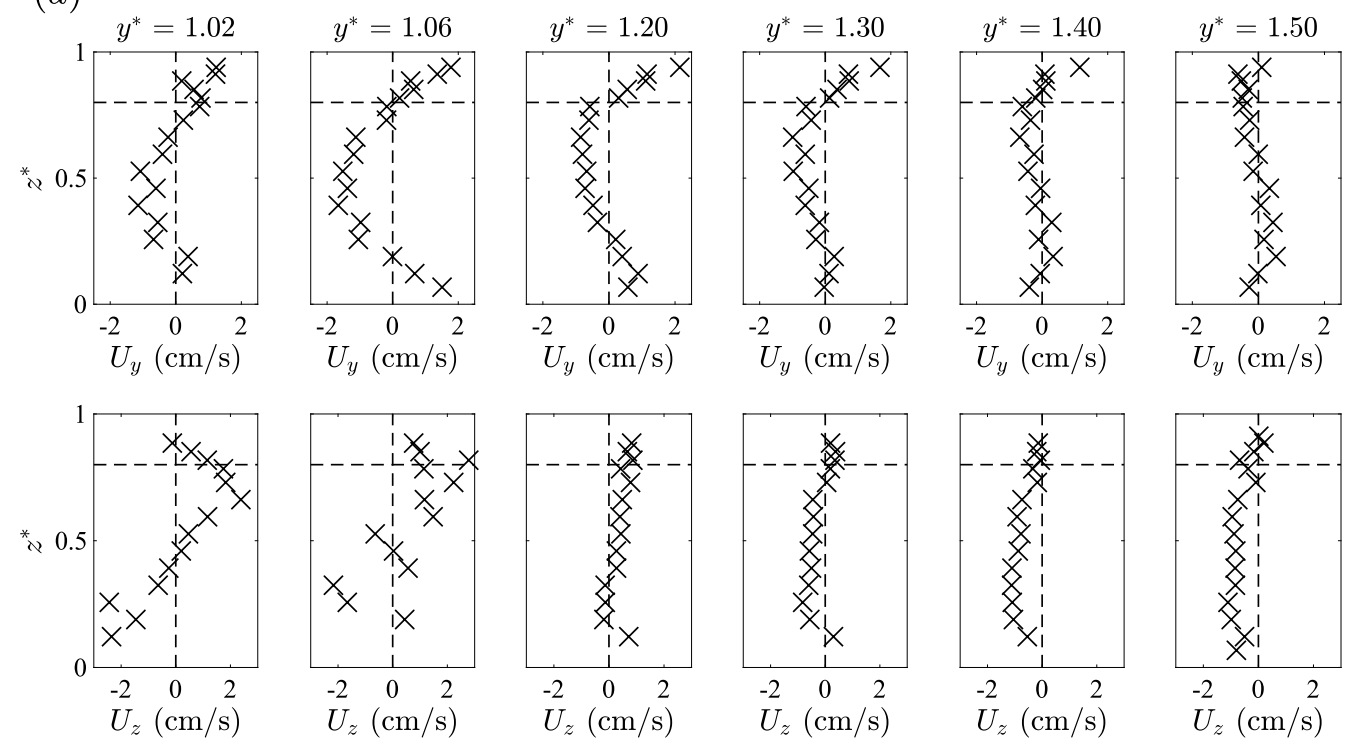

(b)

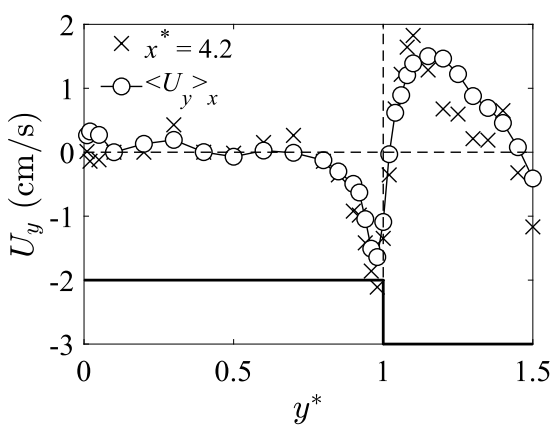

(c)

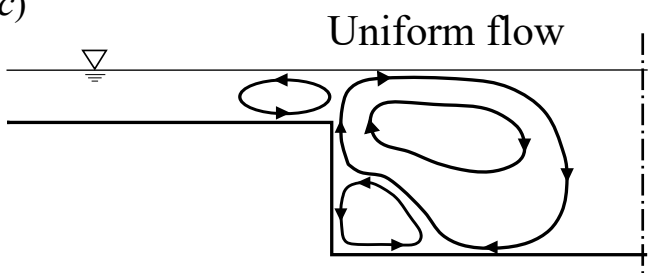

FiguRE 9. Uniform case $(8 \mathrm{~L} / \mathrm{s})$ : (a) vertical distributions of the time-averaged transverse velocity, $U_{y}$, and vertical velocity, $U_{z}$, across a half-MC at $x^{*}=15.9$ (a horizontal dotted line at $z^{*}=0.8$ indicates the bankfull stage in MC); (b) transverse distribution of $U_{y}$ at $z^{*}$ $=0.94$ at $x^{*}=4.2$, and longitudinally averaged values between $x^{*}=4.2$ and 16.8 (denoted $<U_{y}>_{x}$ ); (c) cross-sectional sketch of the three SCs cells at $x^{*}=15.9$ (upstream view). The standard sampling errors in $U_{y}$ and $U_{z}$ are approximately $9 \%$ and $16 \%$, respectively.

from that observed in uniform flow, as shown in Figure 10a for case $20 \mathrm{~L} / \mathrm{s}$ at $x^{*}=4.4$, where the transverse currents are significant (figure $5 \mathrm{a}$ ). The small SC cell revealed for 8 $\mathrm{L} / \mathrm{s}$ (figure 9c) does not emerge at the MC corner. A single SC cell spans over the whole water depth (figures 10a and 10c). This helical motion along $x^{*}$-axis is not related to shear layer turbulence, as KHCSs do not exist at this longitudinal position (figure $3 \mathrm{~b}$, and follow-up figures 14 and 15). This cell is most likely induced by the horizontal shearing between the upper mean flow region above the bank full stage in the MC and the inbank mean flow in the MC. A similar pattern is also found for case $16 \mathrm{~L} / \mathrm{s}$ (not shown here). A horizontal-shearing-induced helical motion was first observed by Shiono \& Muto (1998) in compound meandering channels, then by Bousmar et al. (2004) in compound channels with narrowing FPs, and by Proust et al. (2006) in a compound channel with an abrupt FP contraction. In addition, figure 10b indicates that the longitudinal FP vortex observed in the reference depth-uniform flow (figures $9 \mathrm{~b}-\mathrm{c}$ ), has vanished at $x^{*}=4.4$. 
(a)
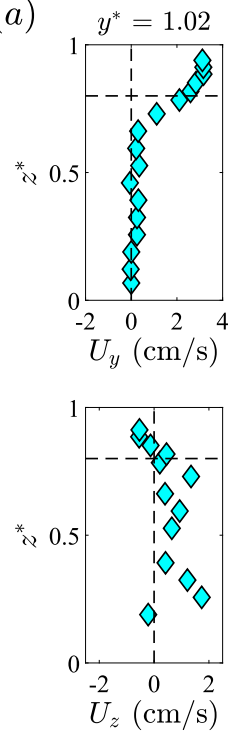
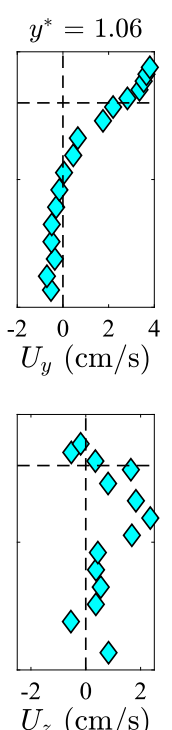
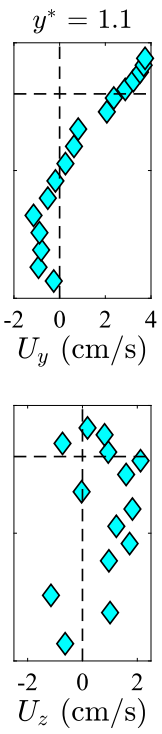
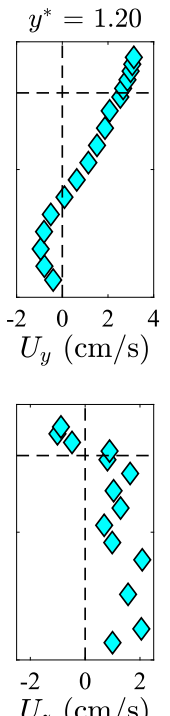
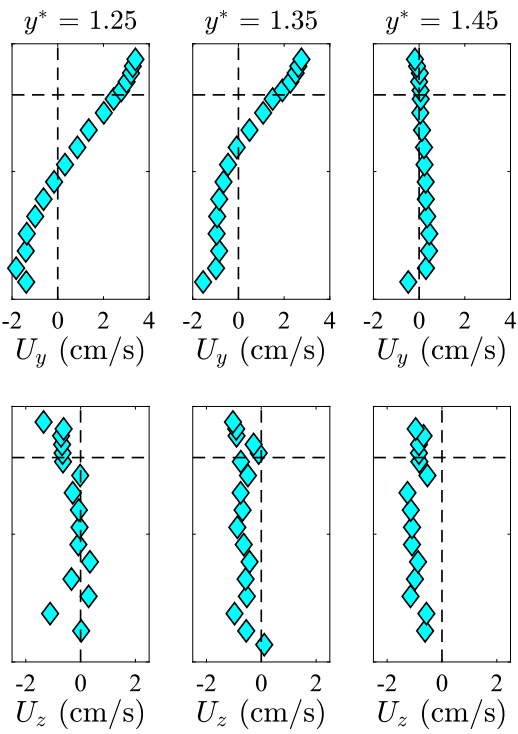

(b)
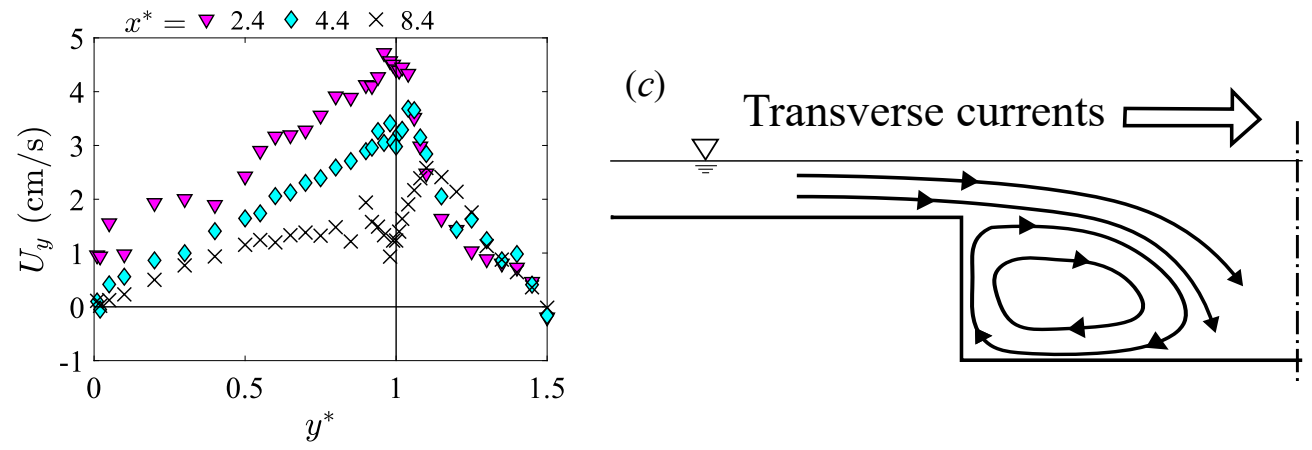

Figure 10. Case $20 \mathrm{~L} / \mathrm{s}$ : (a) vertical distributions of the time-averaged transverse velocity, $U_{y}$, and vertical velocity, $U_{z}$, across a half-MC at $x^{*}=4.4 ;(\mathrm{b})$ transverse distribution of $U_{y}$ at $z^{*}$ $=0.91$ and $x^{*}=2.4,4.4$, and 8.4; (c) sketch of the SC pattern in the half-MC at $x^{*}=4.4$ (upstream view). The standard errors in $U_{y}$ and $U_{z}$ are around $9 \%$ and $16 \%$, respectively.

The SCs across the channel at this position are therefore essentially controlled by the transverse currents directed towards the MC. On the other hand, the $U_{y}$-distribution further downstream at $x^{*}=8.4$ (figure 10b), with a local deficit in $U_{y}$-velocity near the interface on the FP side, indicates the initial stage of the longitudinal FP vortex development.

With transverse currents towards FP, the shapes of the SCs cells are qualitatively comparable to those in the reference flow (figure 9). For case $4 \mathrm{~L} / \mathrm{s}$, vertical profiles of $U_{y}$ and $U_{z}$ at $x^{*}=4.4$ are shown in figure 11a, and figure $11 \mathrm{~b}$ displays transverse distributions of $U_{y}$ at $z^{*}=0.91$ and $x^{*}=2.4,4.4$, and 8.4. From these two figures, we can infer the schematic patterns of the SCs cells drawn in figure 11c. First, water flowing in the lower part of the MC is moving upward and towards the FP (figure 11a). Very close to the interface on the MC side, the $U_{y}$-values are thus essentially negative from either side of the bank full stage $\left(z^{*}=0.8\right)$, highlighting the transverse net mass flux from MC to FP. Second, near the interface in the lower part of the MC, we can infer a small SC cell rotating anti-clockwise when looking upstream, like the corner cell of the uniform 
(a)
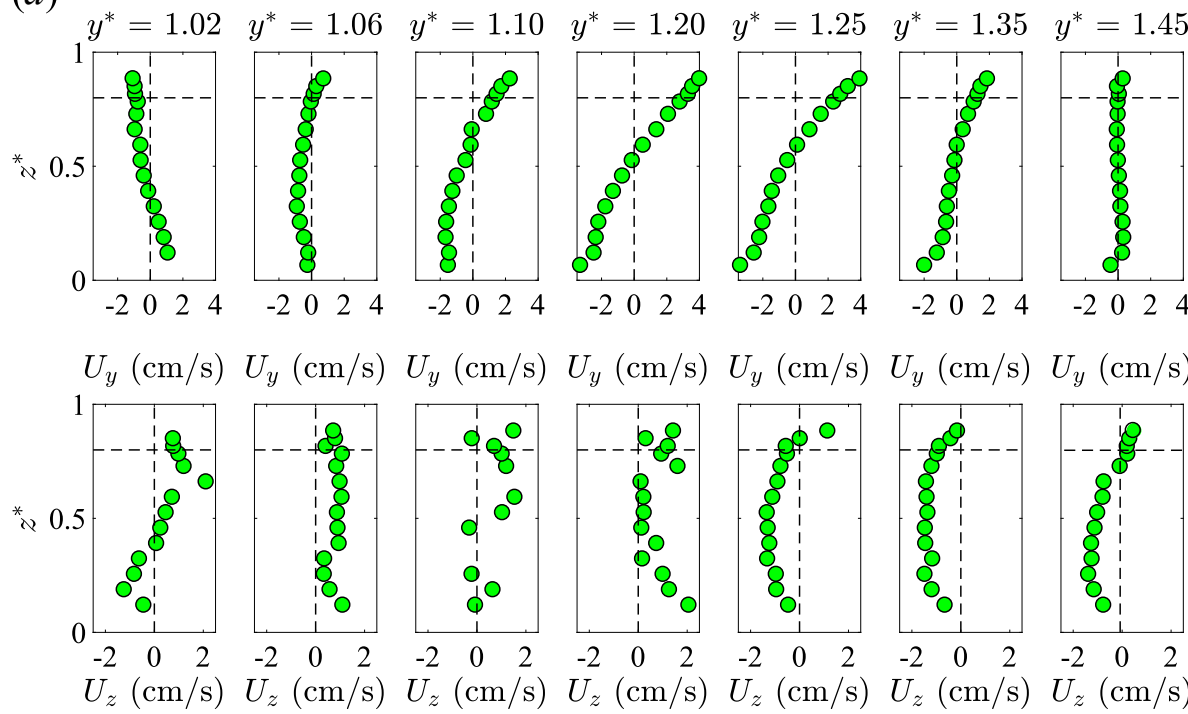

$U_{y}(\mathrm{~cm} / \mathrm{s})$

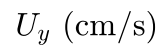

$\begin{array}{llll}-2 & 0 & 2 & 4\end{array}$

(b)

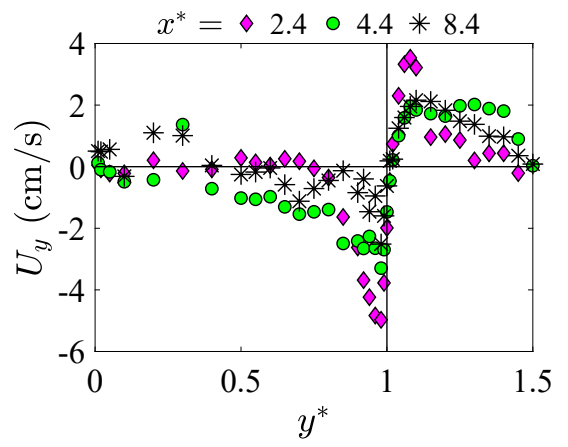

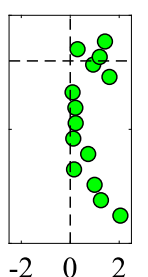
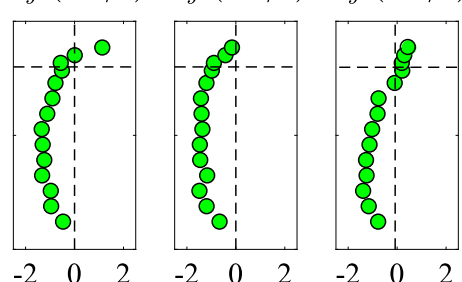

$U_{z}(\mathrm{~cm} / \mathrm{s}) U_{z}(\mathrm{~cm} / \mathrm{s})$

(c)

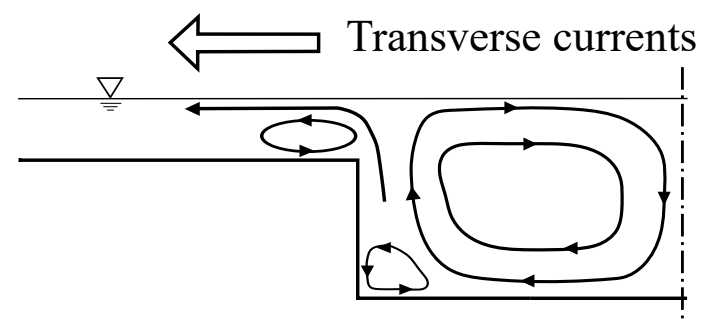

Figure 11. (Colour online) Case $4 \mathrm{~L} / \mathrm{s}$ : (a) vertical distributions of the time-averaged transverse velocity, $U_{y}$, and vertical velocity, $U_{z}$, at $x^{*}=4.4$; (b) transverse distributions of $U_{y}$ at elevation $z^{*}=0.91$ and at various $x^{*}$-positions; and (c) sketch of the SCs patterns in the half-MC at $x^{*}=4.4$ (upstream view). The standard errors in $U_{y}$ and $U_{z}$ are around $9 \%$ and $16 \%$, respectively.

case (figure 9c). Third, when moving away from the interface into MC, a large SCs cell rotates clockwise like the large cell of the uniform case (figure 9c). This cell seems to be bigger than the uniform flow cell, which would be consistent with the increase of the planform shear layer turbulence from $8 \mathrm{~L} / \mathrm{s}$ to $4 \mathrm{~L} / \mathrm{s}$ ( $\S 5$, figure 15). Last, the transverse profiles of $U_{y}$ at $z^{*}=0.91$ (figure 11b) show that the longitudinal FP vortex is fully developed at $x^{*}=8.4$ (compare figure $9 \mathrm{~b}$ and $11 \mathrm{~b}$ ), although its emergence is already visible at the upstream locations $x^{*}=2.4$ and 4.4. A $U_{y}$-profile at $x^{*}=4.4$ measured near the FP bottom (not shown here) and showing some positive $U_{y}$-values near the interface on the FP side confirms the presence of the FP vortex at an early stage of the longitudinal flow development. We can thus conclude that a transverse mean flow from MC to FP occurred above the longitudinal FP vortex, as sketched in figure 11c. 


\section{Kelvin-Helmholtz type coherent structures}

\subsection{Detection using dye tracer}

In a preliminary step, KHCSc in our study were detected with a colored tracer, with an injection point at $x^{*}=6.4$ near the $\mathrm{MC} /$ right $\mathrm{FP}$ interface on the $\mathrm{FP}$ side (see figure 3 for the cases of $4 \mathrm{~L} / \mathrm{s}$ and $20 \mathrm{~L} / \mathrm{s}$ ). For $4 \mathrm{~L} / \mathrm{s}$, the signature of a strong Kelvin-Helmholtz instability downstream of $x^{*}=6.4$ is evident, as weak but noticeable ejections of slow momentum fluid from FP towards the faster flow in the MC can be observed (figure 3a). Each ejection is followed by a strong sweep of high momentum fluid deeply penetrating into the slower flow over the FP. For $20 \mathrm{~L} / \mathrm{s}$ (figure 3b), an instability from either side of the interface is still visible downstream of $x^{*}=6.4$, but its signature is less profound. The fluid ejection from FP to MC is fairly weak, though transverse currents from FP towards MC could have made it easier. This weaker instability cannot be ascribed to the shallowness effects, as observed for shallow mixing layers in non-compound openchannels (e.g., Chu \& Babarutsi 1988; Uijttewaal \& Booij 2000), since the flow depths for $4 \mathrm{~L} / \mathrm{s}$ and $20 \mathrm{~L} / \mathrm{s}$ at $x^{*}=6.4$ were $D_{f}=27.8 \mathrm{~mm}$ and $35.1 \mathrm{~mm}$, respectively (vertical confinement is thus higher for $4 \mathrm{~L} / \mathrm{s}$ where KHCSs are stronger). Note also that the highlighted asymmetry in visualized mixing layers is consistent with the data from mean velocity distributions in figure 8.

\subsection{Characteristic length scales}

To investigate the longitudinal and transverse development of KHCSs, space-time correlations of velocity fluctuations were used, based on the two-point velocity measurements. The employed space-time correlation function is defined as:

$$
R_{i j}^{i}\left(x_{i}, \epsilon_{i}, \tau\right)=\frac{\overline{u_{i}^{\prime}\left(x_{i}, t\right) u_{j}^{\prime}\left(x_{i}+\epsilon_{i}, t+\tau\right)}}{\sqrt{\overline{u_{i}^{\prime 2}\left(x_{i}\right)} \overline{u_{j}^{\prime 2}\left(x_{i}+\epsilon_{i}\right)}}}
$$

where $u_{i}^{\prime}$ is fluctuation of the $i^{\text {th }}$ velocity component, $x_{i}$ is a spatial coordinate (i.e., $x$ or $y), \epsilon_{i}$ is spatial lag in the $x_{i}$-direction, $t$ is time, and $\tau$ is time lag.

The function $R_{i j}^{i}\left(x_{i}, \epsilon_{i}, \tau\right)$ defined by Eq. 5.1 depends on the location of the reference point where a measurement probe is fixed, with another probe moving away point by point. The reference point was always located at the interface, while the second probe was moving laterally in the MC or over the FP, or longitudinally along the interface downstream the flume.

Figure 12a shows examples of spatial correlation function (with zero time lag) of the transverse velocity fluctuation, $u_{y}^{\prime}$, in the streamwise direction along the interface, for three downstream positions of the reference (fixed) upstream probe. An example of the correlation function of $u_{y}^{\prime}$ across the FP at $x^{*}=4.4$ is plotted in figure $12 \mathrm{~b}$ (the shapes of transverse correlation functions at $x^{*}=8.4,12.4$, and 16.4 are similar). Based on these spatial correlation functions, we shall define three characteristic length scales of the KHCSs to be used in our considerations. To reduce potential uncertainties due to the shape of the correlation functions (especially when the two probes are close to each other) and limited maximum lag, we do not use the conventional integral scales and instead define the characteristic scales of the KHCSs as spatial lags corresponding to a particular correlation level (e.g., O’Neill et al. 2004; McDonough 2007).

The first characteristic scale is a streamwise scale along the interface, $\delta_{i n t}^{C S}$, which corresponds to the longitudinal distance between the upstream fixed probe and the downstream moving probe when $R_{y y}^{x}$ crosses zero for the second time (see $\delta_{i n t}^{C S}$ at $x^{*}=14.9$ in figure $12 \mathrm{a}$ ). This scale corresponds, approximately, to the $3 / 4$ of the spacing 

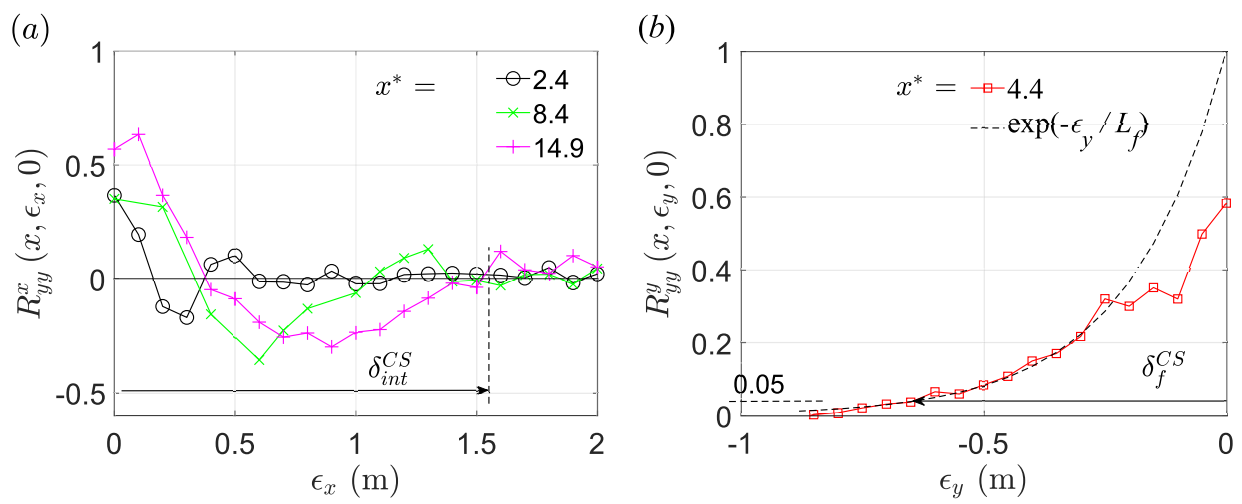

FIGURE 12. Spatial correlation functions of transverse velocity fluctuations, $u_{y}^{\prime}$, for the case of $8 \mathrm{~L} / \mathrm{s}$ at elevation $z^{*}=0.94$ : (a) along the interface $\left(y^{*}=1\right)$, the fixed probe measuring at the $x^{*}$-position (as shown in the legend); and (b) across the right-hand FP, the fixed probe measuring at $y^{*}=1$. Also displayed are the streamwise length scale of KHCSs, $\delta_{i n t}^{C S}$, at $x^{*}=14.9$, and spanwise length scale over the right-hand FP, $\delta_{f}^{C S}$, at $x^{*}=4.4$.

between the dominant structures in the longitudinal direction. The factor of $3 / 4$ follows from the approximation of the streamwise correlation function as a periodic function so that second crossing corresponds to approximately $3 / 4$ of the dominant period in the signal. The second characteristic scale of KHCSs, $\delta_{f}^{C S}$, corresponds to the transverse distance from the interface to the $y^{*}$-value within FP where $R_{y y}^{y}$ reaches 0.05 (figure $12 \mathrm{~b})$. The correlation level of 0.05 is chosen as it represents a level below which the correlation is not distinguishable from zero. Note that this scale is approximately equal to $3 L_{f}$ (Eq. 5.2) if the correlation function is approximated by $\exp \left(-\epsilon_{y} / L_{f}\right)$, with:

$$
L_{f}=\int_{-\infty}^{0} R_{y y}^{y} d \epsilon_{y}
$$

where $L_{f}$ is an integral transverse scale. The exponential approximation of $R_{y y}^{y}$ is found to match data fairly well, as shown in figure $12 \mathrm{~b}$ for the case of $8 \mathrm{~L} / \mathrm{s}$ at $x^{*}=4.4$ within the spatial separations for which the two probes do not have an influence on each other $\left(\left|\epsilon_{y}\right| \geqslant 0.2\right.$, see section 2.4). The third characteristic scale of KHCSs, $\delta_{m}^{C S}$, is similar to $\delta_{f}^{C S}$ but within the MC. The total characteristic width of the KHCSs can thus be defined as a sum of MC-hand and FP-hand scales, i.e.:

$$
\delta^{C S}=\delta_{f}^{C S}+\delta_{m}^{C S}
$$

\subsection{Mismatch between shear layer and KHCSs}

In addition to the above three scales of KHCSs, we can also consider two length scales of the shear layer (based on mean velocity distribution) on either side of the MC/FP interface, following Dupuis et al. (2017a,b). Given the strong asymmetry of the compound open-channel shear layer compared to the conventional free shear layer, these authors have divided the shear layer width, $\delta$, into two parts on either side of the interface:

$$
\delta=\delta_{f}+\delta_{m}
$$

which are defined with 
(a)

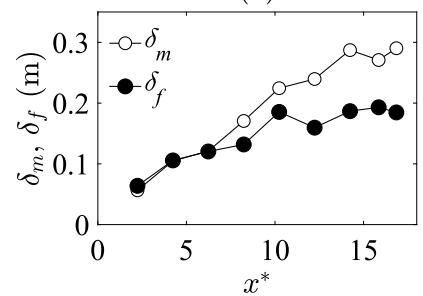

(b)

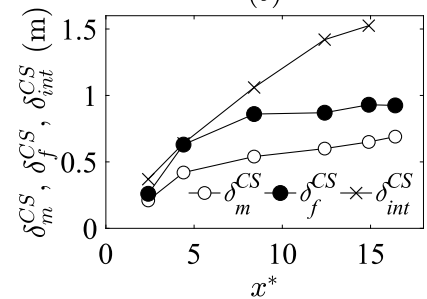

(c)

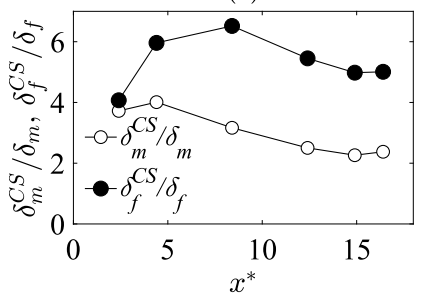

Figure 13. Case $8 \mathrm{~L} / \mathrm{s}$, elevation $z^{*}=0.94$ : (a) shear layer widths based on mean velocity distribution in the MC, $\delta_{m}$, and over the FP, $\delta_{f} ;(\mathrm{b})$ characteristic scales of KHCSs in the MC, $\delta_{m}^{C S}$, in the FP, $\delta_{f}^{C S}$, and along the interface, $\delta_{i n t}^{C S}$; (c) ratios $\delta_{m}^{C S} / \delta_{m}$ and $\delta_{f}^{C S} / \delta_{f}$.

$$
\begin{aligned}
& U_{x}\left(y_{i n t}+\delta_{m} / 2\right)=\frac{U_{x 2}+U_{x, i n t}}{2}, \\
& U_{x}\left(y_{i n t}-\delta_{f} / 2\right)=\frac{U_{x 1}+U_{x, i n t}}{2} .
\end{aligned}
$$

where $y_{\text {int }}$ is the lateral position of the interface. This partition of $\delta$ is based on the assumption that $\delta_{f}$ and $\delta_{m}$ could evolve independently of each other, for both uniform and non-uniform flows (see Dupuis et al. 2017a,b). Representation of the shear layer width by Eqs. 5.4 and 5.5 is similar to Eq. 4.1. However, it explicitly considers the shear layer development on either sides of the interface rather than on either sides of the position of mean velocity $\left(U_{x 1}+U_{x 2}\right) / 2$ as in Eq. 4.1 .

We can now consider the simultaneous evolutions of $\delta_{f}, \delta_{m}, \delta_{f}^{C S}, \delta_{m}^{C S}$, and $\delta_{\text {int }}^{C S}$, which are first shown for the reference depth-uniform flow in figure 13. First, figure 13 highlights a noticeable decoupling of the time-averaged flow with KHCSs all along the flume, as $\delta_{m} \geqslant \delta_{f}$ while $\delta_{m}^{C S} \leqslant \delta_{f}^{C S}$. In addition, figure 13c indicates that, upstream, the growth rate of the structures is higher than that of the shear layer. Further downstream, the opposite trend is observed, and an equilibrium between time-averaged flow and KHCSs is eventually reached at the far end of the flume. Second, figure 13b shows that the transverse extent of the KHCSs is higher over the FP compared to the MC. The assumption that shallowness effects (bed-induced turbulence and flow confinement) on KHCSs are stronger in the FP than in the MC (made in §4.3) thus is not supported with the data. The comparison with non-compound channel shear layers may not be too helpful, as there is no potential decay of destabilizing shear $U_{x 1}-U_{x 2}$ and shear layer turbulence when moving downstream in our case, owing to the presence of the persistent lateral change in topography, which enables the transverse turbulent momentum exchange to be self-sustained.

For the non-uniform flows, the mismatch between the longitudinal developments of $\delta_{m}$ and $\delta_{m}^{C S}$ on the one hand, and between the developments of $\delta_{f}$ and $\delta_{f}^{C S}$ on the other hand, becomes far more visible (figure 14). It is most obvious for $20 \mathrm{~L} / \mathrm{s}$ where KHCSs deeply penetrate into the $\mathrm{FP}$ in the downstream half of the flume $\left(\delta_{f}^{C S}=0.8 \mathrm{~m}\right.$ in the last measuring section), while $\delta_{f} \leqslant 0.05 \mathrm{~m}$ for all $x^{*}$-positions. In the MC, a different trend can be observed where the shear layer starts developing before the KHCSs emergence. The increase in $\delta_{m}$ between $x^{*}=2.4$ and 4.4 (where $\delta_{m}^{C S}=0$ ) is essentially due to the mass and momentum transfers by the time-averaged transverse flow from the FP towards the MC. The same trends are visible for $16 \mathrm{~L} / \mathrm{s}$.

For $12 \mathrm{~L} / \mathrm{s}$, the 'decorrelation' between $\delta_{f}$ and $\delta_{f}^{C S}$ is still significant, as the shear 
(a) $0 \mathrm{~L} / \mathrm{s}$

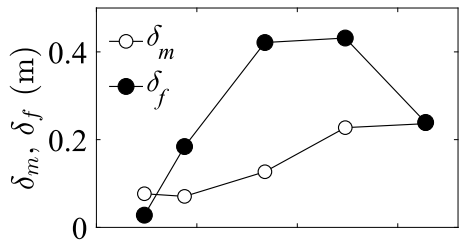

(b) $4 \mathrm{~L} / \mathrm{s}$

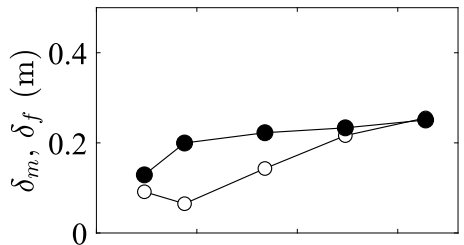

(c) $12 \mathrm{~L} / \mathrm{s}$

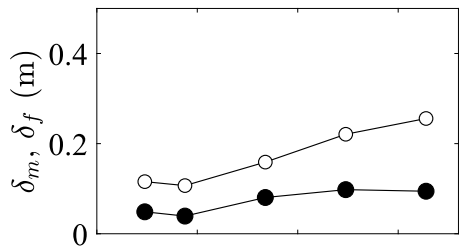

(d) $16 \mathrm{~L} / \mathrm{s}$

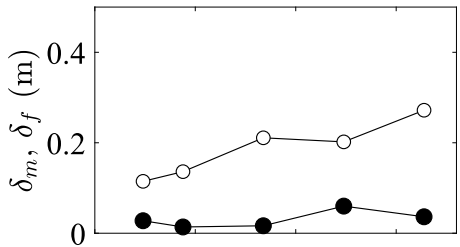

(e) $20 \mathrm{~L} / \mathrm{s}$

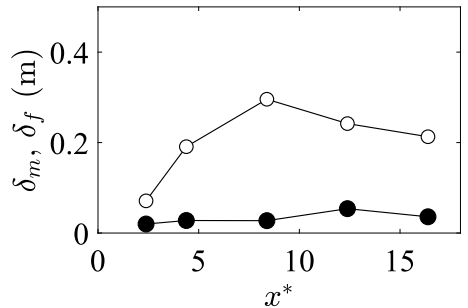

(f) $0 \mathrm{~L} / \mathrm{s}$

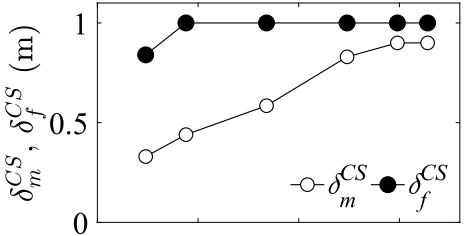

(g) $4 \mathrm{~L} / \mathrm{s}$

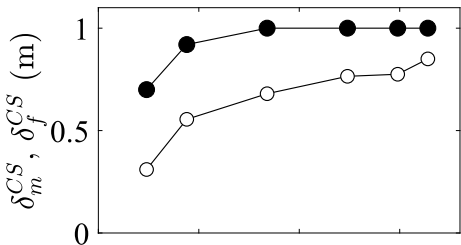

(h) $12 \mathrm{~L} / \mathrm{s}$

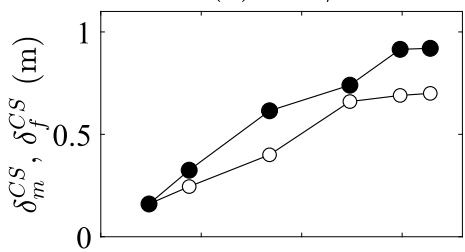

(i) $16 \mathrm{~L} / \mathrm{s}$

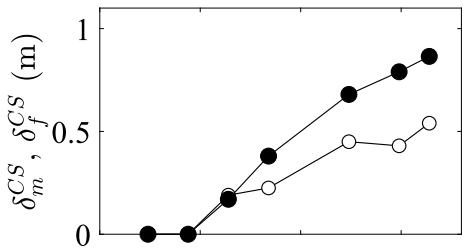

(j) $20 \mathrm{~L} / \mathrm{s}$

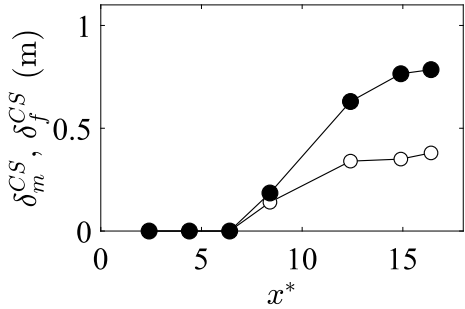

FIGURE 14. Non-uniform cases: (a-e) shear layer widths based on the mean velocity distributions in $\mathrm{MC}, \delta_{m}$, and over $\mathrm{FP}, \delta_{f}$; (f-j) characteristic scales of KHCSs in MC, $\delta_{m}^{C S}$, and in $\mathrm{FP}, \delta_{f}^{C S}$.

layer is displaced in the MC by the transverse currents. In the $\mathrm{MC}$, we may assume that KHCSs contribute to the shear layer development with a ratio $\delta_{m}^{C S} / \delta_{m}$ ranging from 3 to 2.5 in the second half of the flume, close to the values observed for the uniform case (figure 13c).

For 0 and $4 \mathrm{~L} / \mathrm{s}$, a noticeable difference with the previous cases can be observed. The FP flow is accelerated by the KHCSs but also by the time-averaged flow as highmomentum fluid from the MC is entering the FP (see the values of $-\rho U_{x d} U_{y d}$ in figure 5f). Both the mean flow and the KHCSs therefore contribute to the increase in $\delta_{f}$, as also observed in diverging FPs (e.g., Proust et al. 2010). The difference in the relaxation 
(a)

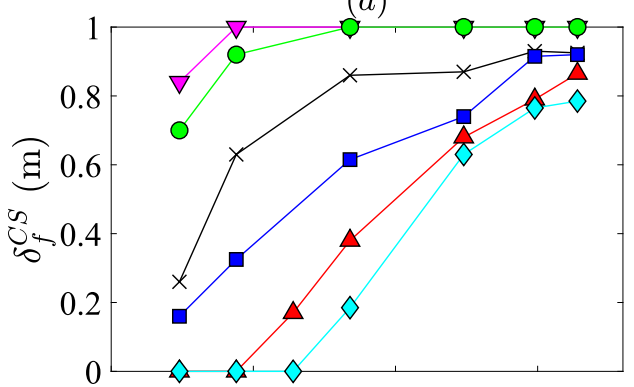

(b)

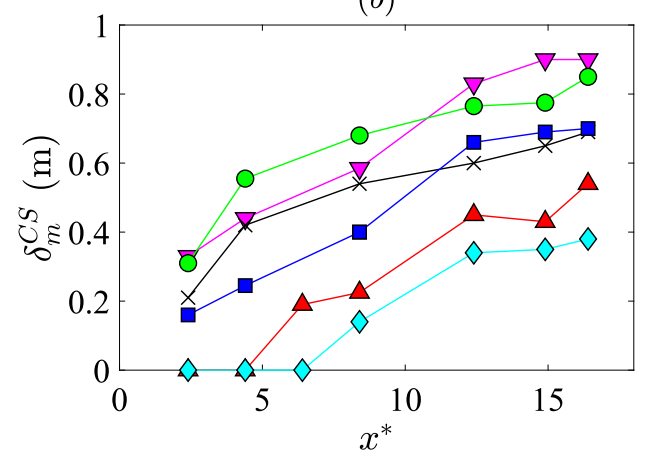

$(c)$

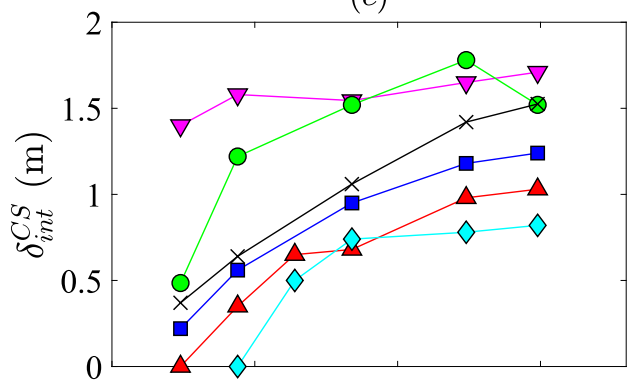

$(d)$

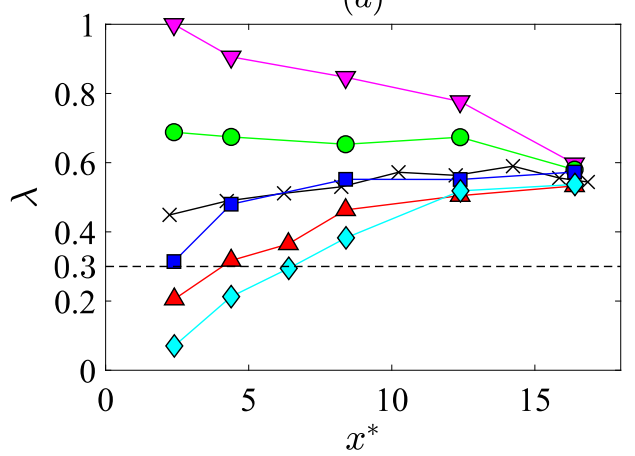

FIGURE 15. Characteristic scales of KHCSs at $z^{*}=0.94(a)$ in FP, $\delta_{f}^{C S},(b)$ in MC, $\delta_{m}^{C S}$, and $(c)$ along the interface, $\delta_{i n t}^{C S}$, and $(d)$ dimensionless shear $\lambda$ (see Eq. 5.6), for the cases of $0 \mathrm{~L} / \mathrm{s}(\nabla), 4 \mathrm{~L} / \mathrm{s}(\circ), 8 \mathrm{~L} / \mathrm{s}(\times), 12 \mathrm{~L} / \mathrm{s}(\square), 16 \mathrm{~L} / \mathrm{s}(\triangle)$, and $20 \mathrm{~L} / \mathrm{s}(\diamond)$.

towards flow uniformity (depending on the direction of transverse currents) visible in figure 8 partly originates from noted trends in KHCSs developments and the effect of the depth-averaged transverse mean flow (§7.2). Last, we can observe a spatial delay between the developments of KHCSs and shear layer near the flume entrance, similar to the uniform flow (figure 13c). Large KHCSs extend nearly over the entire FP width within the upstream part of the flume, which is not matched by the shear layer lateral extent.

It is important to notice that the KHCSs length scale in MC, $\delta_{m}^{C S}$, can exceed the half width of the MC $(0.5 \mathrm{~m})$. This feature is observed for both uniform (figure 13b) and non-uniform flows (figures 14f-h). The MC centreline is a symmetry axis where gradient $d U_{x} / d y$, time-averaged transverse flow and associated momentum transfer are zero. This results in a shear layer width in MC, $\delta_{m}$, always less than $0.5 \mathrm{~m}$ (figures 13 and 14). On the other hand, the MC center-line is not a barier for the structures of the instantaneous velocity field, such as KHCSs, and dye tracer, which can cross $y^{*}=0.5$ intermittently, directed from one bank to another, resulting in $\delta_{m}^{C S} \geqslant 0.5 \mathrm{~m}$. Note that vortices wider than a half width of MC were also observed by Stocchino \& Brocchini (2010, figure 3). It is also worth highlighting at this point that $\delta_{m}^{C S}$ is a scale, not an actual size, and that this scale is three times larger than the integral transverse scale (as explained in section $5.2)$.

\subsection{Effect of the dimensionless velocity shear on KHCSs}

The longitudinal developments of $\delta_{f}^{C S}, \delta_{m}^{C S}$, and $\delta_{i n t}^{C S}$ for all cases are grouped together in figures 15a-c. First important feature to note is a wide range of the characteristic scales of KHCSs at a given $x^{*}$-position across the flow cases. In particular, at the 
first measuring section the length scales systematically increase with increase in the destabilizing shear $U_{x 2}-U_{x 1}$ (table 1) or dimensionless shear $\lambda$ (Brown \& Roshko 1974):

$$
\lambda=\frac{U_{x 2}-U_{x 1}}{U_{x 2}+U_{x 1}}
$$

which is plotted in figure $15 \mathrm{~d}$. It is useful to mention at this point that the dimensionless shear $\lambda$ can be physically interpreted in a number of ways. One of them is to consider $\lambda$ as a measure of a ratio of the conventional vorticity scale $\delta_{\omega}=\left(U_{x 2}-U_{x 1}\right) /\left(d U_{x} / d y\right)_{\text {int }}$ to the shear scale $\delta_{s}=U_{x, \text { int }} /\left(d U_{x} / d y\right)_{\text {int }} \approx 0.5\left(U_{x 2}+U_{x 1}\right) /\left(d U_{x} / d y\right)_{\text {int }}$, i.e., $\lambda=0.5 \delta_{\omega} / \delta_{s}$. It can also be viewed as a square root of a ratio of the turbulence energy associated with KHCSs $\left(\propto\left(U_{x 2}-U_{x 1}\right)^{2}\right)$ to the energy associated with bedfriction depth-scale turbulence $\left(\propto\left(0.5\left(U_{x 2}+U_{x 1}\right)\right)^{2}\right)$. Interestingly, our data show that KHCSs scales increase with reduction of the flow depth (i.e., with an increase in flow confinement) from $20 \mathrm{~L} / \mathrm{s}$ to $0 \mathrm{~L} / \mathrm{s}$ (table 1 ), in contrast to observations for non-compound open-channels mentioned earlier.

Second, KHCSs are observed along the whole measuring domain for 0, 4, 8 and 12 $\mathrm{L} / \mathrm{s}$, but not for 16 and $20 \mathrm{~L} / \mathrm{s}$. For these two cases, KHCSs are absent close to the flume entrance (at $x^{*}=2.4$ ), although neither destabilizing shear $U_{x 2}-U_{x 1}$ (table 1 ) nor dimensionless shear $\lambda$ (figure 15d) are zero there.

The emergence of KHCSs is found to be related to the local (threshold) value of the dimensionless shear $\lambda$ (or $\left.\delta_{\omega} / \delta_{s}\right)$. This result, based on two-point measurements, is fully consistent with earlier one-point measurements of Proust et al. (2017), who investigated 25 flow cases in two different open-channel flumes at three levels of vertical flow confinement. A dimensionless shear $\lambda$ higher than 0.3 was found to be a necessary condition for the emergence and development of the KHCSs. Note that as $\lambda$ is a monotonically decreasing function of velocity ratio $U_{x 1} / U_{x 2}$, the condition $\lambda \geqslant 0.3$ is equivalent to $U_{x 1} / U_{x 2} \leqslant \approx 0.5$. No effect of flow confinement on the threshold value of $\lambda$ was noted. In the present study (figure 15), no KHCS can be observed if $\lambda<0.3$, in agreement with the previous findings of Proust et al. (2017). When the dimensionless shear reaches 0.3, KHCSs start emerging along the interface before expanding into MC and FP (see $\delta_{f}^{C S}, \delta_{m}^{C S}$, and $\delta_{\text {int }}^{C S}$ for 16 and $20 \mathrm{~L} / \mathrm{s}$, figure 15). This is also clearly seen in figure $3 \mathrm{~b}$ for $20 \mathrm{~L} / \mathrm{s}$, where the instability starts developing longitudinally along the interface downstream of $x^{*}=6.4$ with a very small amplitude in the lateral direction (longitudinal oscillation without formation of spiral-shaped vortices, as sketched by Lesieur (2013, p. 50) for free mixing layers).

The conditions $\lambda \geqslant 0.3$ and $U_{x 1} / U_{x 2} \leqslant \approx 0.5$ are also equivalent to:

$$
\delta_{\omega} \geqslant 0.6 \delta_{s}
$$

demonstrating that KHCSs emerge once the vorticity strength at the inflection point (quantified with $\delta_{\omega}$ ) exceeds the effect of velocity shear (quantified with $\delta_{s}$ that characterizes interplay between mean energy diffusion and conversion of mean energy to turbulence). Recalling that the dimensionless shear $\lambda$ represents a square root of the ratio of the turbulence energy associated with KHCSs to the energy associated with bed-friction depth-scale turbulence one may argue that KHCSs start to emerge once large-scale energy becomes dominant in the velocity spectrum.

The criterion of $\lambda \geqslant 0.3$ for the emergence of KHCSs was recently found to be confirmed in the experiments of Caroppi et al. (2019) that focused on water-vegetation interface in open-channel flow. On the other hand, the experiments of Akutina et al. (2019) show that in the case of a lateral bed-roughness variation in shallow open-channel flow with 

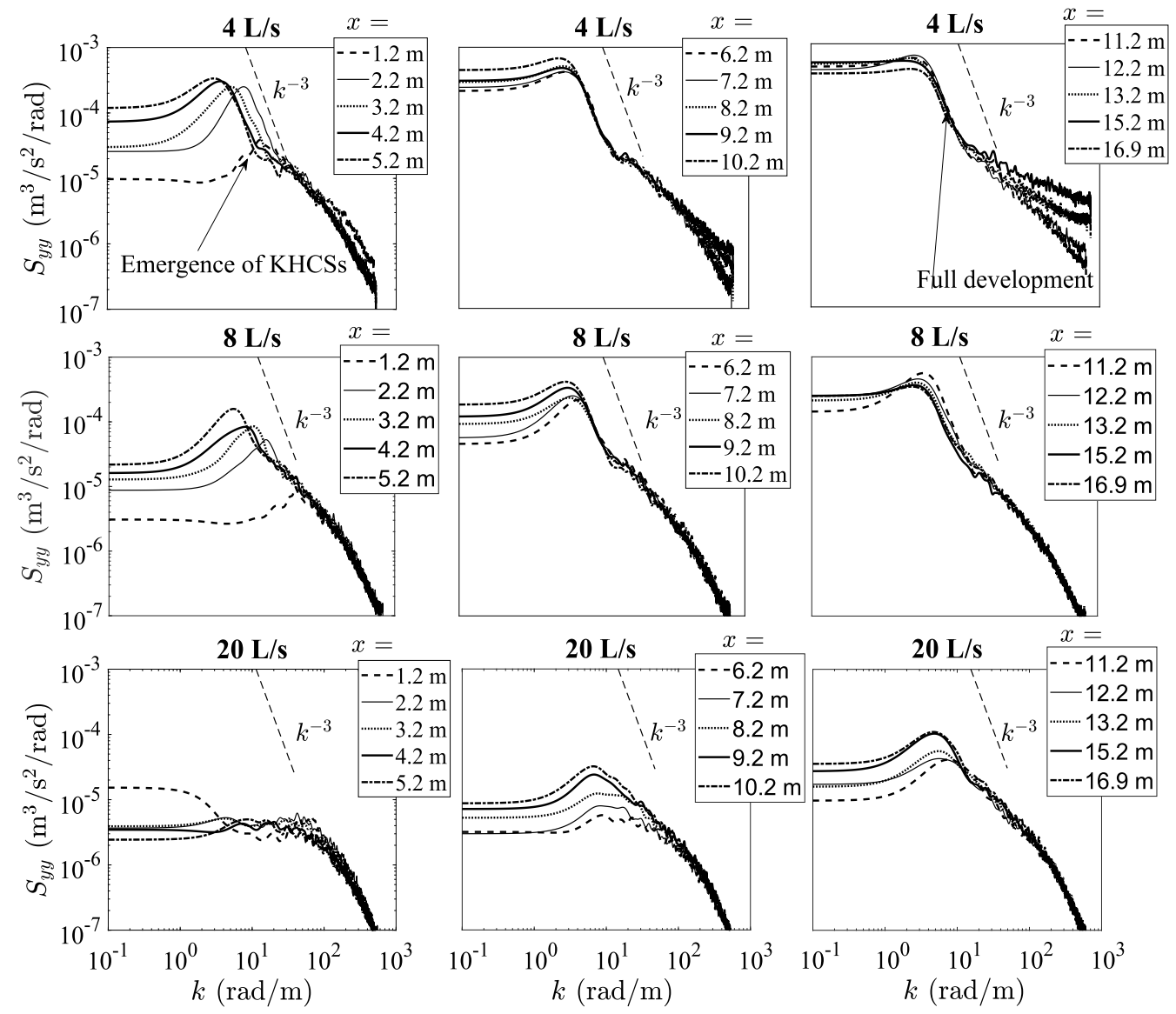

Figure 16. Power density $S_{y y}$ of transverse velocity fluctuations $u_{y}^{\prime}$, as a function of wavenumber $k$, along the interface at elevation $z^{*}=0.91$ for the cases of 4,8 , and $20 \mathrm{~L} / \mathrm{s}$.

very low submergence, this criterion is no more valid. This might be due to the effect of the bed-induced strong turbulence generated at the top of the roughness elements that would prevent emergence of the transverse turbulent motions in the case of a very low submergence of the roughness elements.

Third, figures 15a to 15c highlight, for all flow cases, an increase of the KHCSs size in the transverse direction (both in $\mathrm{MC}$ and $\mathrm{FP}$ ) and in the longitudinal direction, when moving downstream. In MC, the development rate of KHCSs does not seem to be sensitive to the magnitude and direction of transverse currents, as the growth rate $d\left(\delta_{m}^{C S}\right) / d x$ does not significantly change from one case to another. Over the FP, the evolution of $\delta_{f}^{C S}$ appears to be more controlled by the location (downstream position $x^{*}$ ) where KHCSs emerge, i.e., where $\lambda$ exceeds 0.3 . The same applies for the growth of $\delta_{\text {int }}^{C S}$ along $x^{*}$. We can thus conclude that, unlike the shear layer, KHCSs are relatively weakly influenced by the transverse currents.

\subsection{KHCSs and power density spectra of velocity fluctuations}

In addition to using colored tracer and two-point velocity measurements to detect KHCSs, we also relied on power density spectra of velocity fluctuations. Figure 16 shows the power density $S_{y y}$ of the transverse velocity fluctuation $u_{y}^{\prime}$ as a function of 
wavenumber $k$ along the interface, for three flow cases. The wavenumber spectra are obtained from the frequency spectra assuming the applicability of the frozen turbulence hypothesis and using local time-averaged velocity as a convection velocity (with $k=$ $2 \pi f / U_{x}$ and $f=$ frequency). The spectral evolution is characterized by the appearance of a sharp bump at large scales, which is then flanked by a $k^{-3}$ spectrum (on the righthand side of the bump) when moving downstream. A similar spectral behaviour was first observed in $2 \mathrm{D}$ turbulence inside a box, in the so-called condensation regime in which the system boundaries impose the peak vorticity value (see e.g., Tabeling 2002, figure $56)$. We may speculate that the $k^{-3}$ scaling range in the observed spectra may reflect an enstrophy cascade (e.g., in freely decaying turbulence the theory of Kraichnan (1967) and Batchelor (1969) predicted an energy spectrum in the form $\left.E(k) \approx t^{-2} k^{-3}\right)$.

In the present case, instead of a turbulence decay, we observe a growth of the $k^{-3}$ spectrum when moving downstream, as the shear layer turbulence is self-sustained by the lateral change in topography. The wavenumber corresponding to the sharp bump is most likely related to the streamwise length scale of the KHCSs, $\delta_{\text {int }}^{C S}$. For instance, for $8 \mathrm{~L} / \mathrm{s}$, an equilibrium state is reached with an energy peak at $k \approx 3 \mathrm{rad} / \mathrm{m}$, i.e., with a wavelength of approximately $2 \mathrm{~m}$, which is consistent with $\delta_{\text {int }}=1.5 \mathrm{~m}(3 / 4$ of the distance between two vortex cores) at $x^{*}=15$ in figure $15 \mathrm{c}$.

The present experimental data also demonstrate that the appearance of the local peak of $S_{y y}$ within the intermediate range of wavenumbers (figure 16) always corresponds to the emergence of KHCSs. For instance, for $20 \mathrm{~L} / \mathrm{s}$, KHCSs start growing downstream at $x^{*}=6.4$ (figures $3 \mathrm{~b}$ and $15 \mathrm{c}$ ), while a small local peak in $S_{y y}$ appears between $x^{*}=6.2$ and 7.2 (figure 16). For $4 \mathrm{~L} / \mathrm{s}$ and $8 \mathrm{~L} / \mathrm{s}$, the local peak in $S_{y y}$ is visible at $x^{*}=1.2$ being consistent with the early emergence of KHCSs noted in the previous section (figure 15).

The power density spectra thus enable to detect both the location of the emergence of KHCSs and the location where these structures become fully developed. For 4 and $8 \mathrm{~L} / \mathrm{s}$, a noticeable range of $k^{-3}$ can be observed already at $x^{*} \geqslant 2.2$, consistent with the results for the characteristics scales (figure 15), since the KHCSs are already very well developed in the upper part of the flume for these flows. At the interface, this results in high values of the transverse Reynolds shear stress, transverse and longitudinal turbulence intensities, as shown in figures $5 \mathrm{c}, 5 \mathrm{~d}$, and $5 \mathrm{e}$, respectively. The spectra in figure 16 indicate that a fairly long distance can separate the locations of emergence and full development with fully established -3 scaling range; e.g., for $4 \mathrm{~L} / \mathrm{s}$ and $8 \mathrm{~L} / \mathrm{s}$ (figure 16), these locations are respectively located at $x^{*}=1.2$ and 12.2 , while for $12 \mathrm{~L} / \mathrm{s}$ (not shown here) at $x^{*}=2.2$ and $x^{*}=11.2$. For $20 \mathrm{~L} / \mathrm{s}$, the $k^{-3}$ spectrum is hardly visible even at $x^{*}=16.9$, owing to the late emergence of the KHCS $\left(\right.$ at $\left.x^{*} \approx 6\right)$.

Finally, it is worth mentioning that the spectra with a distinct bump and associated $-3 k$-range have been observed in open-channel flows for various contexts, in which quasi2D turbulence co-exists with 3D turbulence. It was observed e.g., by Dracos et al. (1992) in shallow turbulent jets, by Uijttewaal \& Booij (2000) in mixing layers in non-compound open-channel flows, and by Stocchino \& Brocchini (2010) and Proust et al. (2017) in compound open-channel flows. However, the solid link between the $k^{-3}$ spectrum of pure 2D turbulence (without vortex stretching, based on 2D Navier-Stokes equations) and the $k^{-3}$ spectra observed in quasi-2D turbulence has not been yet fully established theoretically. 
Floodplain (FP)

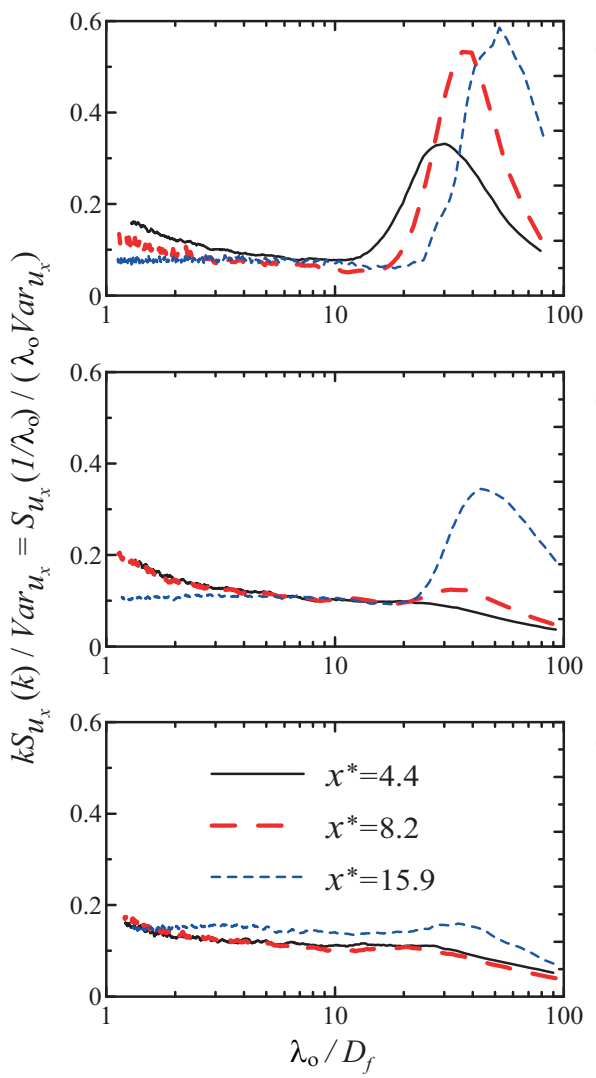

Main channel (MC)

(a)

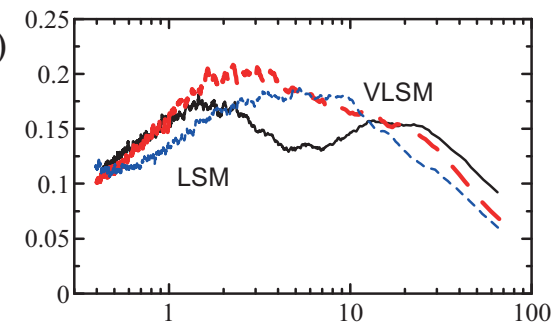

(b)

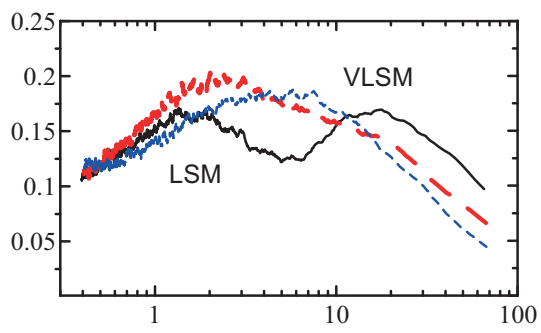

(c)

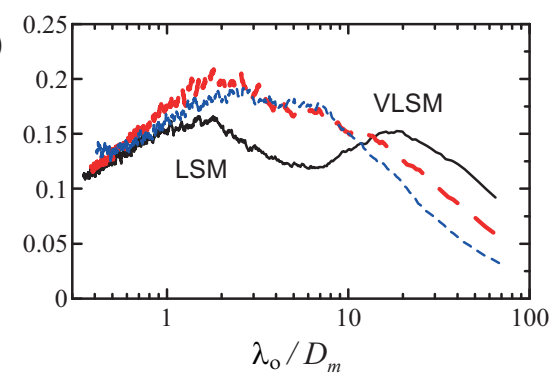

FiguRE 17. One-dimensional pre-multiplied spectra of streamvise velocity fluctuations for flow cases: $4 \mathrm{~L} / \mathrm{s}(\mathrm{a}) ; 8 \mathrm{~L} / \mathrm{s}(\mathrm{b})$; and $16 \mathrm{~L} / \mathrm{s}(\mathrm{c}) . \lambda_{0}$ is wavelength of a spectral component; Var $u_{x}$ is variance of the streamwise velocity.

\section{Very-large-scale motions}

\subsection{Uniform flow}

It is currently widely accepted that the flow structure in straight uniform compound channels represents an interplay of the dynamic effects due to velocity shear in transverse and vertical directions, involving at least three momentum transfer mechanisms: (1) topography-induced SCs which are time-averaged streamwise helical motions; (2) planform (or horizontal) turbulence, which is typically manifested as large coherent structures (KHCSs) emerging as a result of Kelvin-Helmholtz instability due to the inflection point in the transverse velocity profile at the MC/FP interface; and (3) bedgenerated turbulence that shares (at least conceptually) many features with that observed in boundary layers and pipes (e.g., Nezu et al. (1999); Ikeda \& McEwan (2009)).

In conventional uniform open-channel flow with rectangular cross-section, the bedgenerated turbulence may consist of large-scale motions (LSMs, up to 3-4 flow depths long) and very large scale motions (VLSMs, up to 50 flow depths long or even longer), as has been highlighted by Adrian \& Marusic (2012) and recently demonstrated in high-precision experiments by Cameron et al. (2017). The data for this flow type show that VLSMs contributions to the total turbulence energy and turbulent shear stresses may reach up to 50\% (Cameron et al. 2017, 2019) and, thus, these motions should be 
considered as a dominant flow feature. It was also shown that open-channel VLSMs play critical roles in sediment dynamics and mixing (Cameron et al. 2019). The interactions of VLSMs with other flow features such as SCs and/or planform turbulence (e.g., KHCSs) remain unclear. Indeed, transverse flow heterogeneity due to the roughness change or topographical change may affect the appearance and strength of VLSMs, as has been recently shown for the case of a rectangular channel with a bed covered by streamwise ridges (Zampiron et al. 2019; Zampiron 2019). At spanwise spacing between ridges less than two flow depths the VLSMs are completely suppressed, presumably by strong SCs induced by the ridges. Although SCs and VLSMs share some common features such as helical motion patterns, there are some significant differences. On the one hand, VLSMs are represented by pairs of instantaneous meandering counter-rotating helical motions which are typically not seen in the time-averaged velocity fields. The SCs, in turn, do not need VLSMs to exist as SCs are generated by the action of turbulence heterogeneity and anisotropy.

There has been no information reported regarding emergence and significance of VLSMs in straight compound channels and thus our long-term (7 hrs) velocity measurements may provide some preliminary insights. To identify the existence of VLSMs in our experiments we used the pre-multiplied one-dimensional wavenumber spectra of streamwise velocity fluctuations that proved to be the tool of choice for this task (e.g., Kim \& Adrian 1999; Hutchins \& Marusic 2007; Cameron et al. 2017). As we measured velocity time series at fixed spatial locations, the obtained frequency spectra have been transformed into wavenumber spectra by employing Taylor's frozen turbulence hypothesis with local time-averaged velocity as the convection velocity.

Before discussing the pre-multiplied spectra we would like to mention that for VLSMs to be observed in a laboratory flume one needs a sufficient development length for them to emerge and fully establish. Considering conventional open-channel-flow with rectangular cross-section, Zampiron (2019) has recently demonstrated that the minimum flow lengths required for the bulk statistics (mean velocity, variance, skewness and kurtosis), SCs, and LSMs to fully establish need to be between 50 and 70 flow depths. The development distance for VLSMs appeared to be even longer. These results should be treated as suggestive only as they are likely to depend on specific experimental conditions such as flow aspect ratio, relative submergence, roughness geometry, channel shape, and other parameters. Nevertheless, using Zampiron's (2019) values as indicators we can assess the stage of the flow development in terms of LSM and VLSM for all three locations of longterm measurements $\left(x^{*}=4.4,8.2\right.$, and 15.9). Over FP, the closest measuring location (4.4 $\mathrm{m}$ ) is approximately 145 flow depths from the flume entrance and thus we could expect that flow is already fully developed there, if the potential effects of the transverse shear layer and KHCSs are excluded from consideration. Over MC, however, the situation is different: Zampiron's (2019) conditions would be reasonably met only at the downstream measurement location at $x^{*}=15.9$ which is equivalent to $106 x / D_{m}$, and probably at $x^{*}=8.2$ that corresponds to $55 x / D_{m}$. Our experimental data for compound channel flows, however, do not support these expectations.

Figure 17 shows pre-multiplied spectra of streamwise velocity for all locations of longterm measurements (left-hand column is for FP and right-hand column is for MC). The smallest scales shown in these plots are defined by the size of the ADV sampling volume and its effect on the recorded velocities. Starting with MC, we first note that at the upstream location $\left(x^{*}=4.4, x / D_{m}=30\right)$ the pre-multiplied spectrum exhibits a shape typical for the conventional flow in a rectangular channel (Cameron et al. 2017), i.e., it includes two 'hills' that most likely reflect the presence of LSMs (with wavelength $\lambda_{0} \approx 1.6 D_{m}$ ) and VLSMs (with $\lambda_{0} \approx 18 D_{m}$ ), figure $17 \mathrm{~b}$, right-hand plot. This comes 
as a surprise bearing in mind a relatively short distance from the flume entrance $\left(x / D_{m}\right.$ $=30$ ), which is appreciably less than the development lengths of 50 to 70 flow depths in a conventional rectangular flume (Zampiron 2019). A possible explanation of this discrepancy may relate to the use of the flow depth $D_{m}$ as a scale, which may not be appropriate for our experimental set up. An alternative scaling of the streamwise coordinate with the distance of the measurement point from the bed gives a relative distance from the flume entrance around 150, which seems sufficient for the emergence of VLSMs. The pre-multiplied spectrum for the transverse velocity component does not show a peak at $\lambda_{0} \approx 18 D_{m}$ while the coherence function between streamwise and spanwise velocity components do not reveal any correlations at the scales of LSMs $\left(\lambda_{0} \approx 1.6 D_{m}\right)$ and VLSMs $\left(\lambda_{0} \approx 18 D_{m}\right)$. These observations are consistent with Cameron et al. (2017) for a conventional open-channel flow and thus they exclude potential relation of the spectral hills to KHCSs or other transverse shear layer effects. Moving downstream to $x / D_{m}=55$, the spectrum changes its shape, with the LSM hill growing and moving towards larger scales while the VLSM hill 'dissolving'. At the most downstream location $\left(x / D_{m}=106\right)$, the spectrum completely losses its bi-modal shape, transforming in a bell-shaped spectrum with a maximum at 4-5 flow depths. The observed downstream evolution of the pre-multiplied spectrum most likely reflects combined dynamic effects of the transverse shear layer, SCs, and KHCSs. The data suggest that these flow features were insufficiently developed in the near-bed region of $\mathrm{MC}$ at $x^{*}=4.4$ allowing emergence of both LSMs and VLSMs at this location. However, further downstream their effects become sufficiently strong to suppress VLSMs, similar to the effect of SCs that suppressed VLSMs in the study of Zampiron et al. (2019).

Turning to FP, we note no visible LSM hills within the smaller scale range of the pre-multiplied spectra of streamwise velocity at all three studied locations (figure 17b, left-hand plot). Their absence is probably due to the mutual effects of the measurement noise and insufficient spatial resolution as the ADV sampling volume is comparable to the FP flow depth. Focusing on the spectrum at larger scales we note that although at $x^{*}=4.4$ there is no visible spectral hill, it does appear at $x^{*}=8.2\left(\right.$ around $\lambda_{0} / D_{f}=$ 40 ), becoming a dominant spectral feature at $x^{*}=15.9$ (around $\lambda_{0} / D_{f}=50$ ). The premultiplied spectra of the transverse velocity exhibit similar hills at the same wavelengths, which combined with the high coherence between streamwise and transverse velocities at these wavelengths (not shown here) suggest that their origin relates to the KHCSs that introduce strong periodicity in both streamwise and spanwise velocities over FP. The wavelengths $\lambda_{0} / D_{f}=40\left(\right.$ at $\left.x^{*}=8.2\right)$ and $\lambda_{0} / D_{f}=50\left(\right.$ at $\left.x^{*}=15.9\right)$ are in complete agreement with the correlation lengths $\delta_{\text {int }}^{C S}$ shown in figures $13 \mathrm{~b}$ and $15 \mathrm{c}$ (recall that $\delta_{\text {int }}^{C S}$ corresponds to the 0.75 of the dominant length scale, $\S 5.2)$.

Overall, the data for the uniform flow case $(8 \mathrm{~L} / \mathrm{s})$ suggest that in the initial development of KHCSs, SCs, LSMs and VLSMs in MC, the latter two appear to be fairly competitive but then they are quickly suppressed by the effects of either the transverse shear layer, KHCSs or SCs, or their combined work. At the same time, it seems that within FP the effects of KHCSs became already dominant at $x^{*}=4.4$ suppressing the conditions allowing VLSMs to form. The dominating effect of KHCSs is likely supplemented by the artificial grass on the FP bed that could help to prevent emergence of VLSMs. The data indicate that the transverse shear layer, KHCSs and SCs become the most dominant features of the flow dynamics shortly after flow enters the flume.

\subsection{Effects of the transverse currents}

The effects of the transverse currents on LSMs and VLSMs within MC and FP can be seen in figures $17 \mathrm{a}(4 \mathrm{~L} / \mathrm{s})$ and $17 \mathrm{c}(16 \mathrm{~L} / \mathrm{s})$. Compared to the benchmark case of 
$8 \mathrm{~L} / \mathrm{s}$ (figure 17b) discussed in $\S 6.1$, the pre-multiplied spectra in MC for both $4 \mathrm{~L} / \mathrm{s}$ and $16 \mathrm{~L} / \mathrm{s}$ appear to be very similar in shape and magnitudes. This observation suggests that near-bed turbulence and its evolution along the flow are only weakly (if at all) influenced by the transverse currents. The situation within FP is dramatically different. As in $8 \mathrm{~L} / \mathrm{s}$ case, no signatures of VLSMs emerge in $4 \mathrm{~L} / \mathrm{s}$ and $16 \mathrm{~L} / \mathrm{s}$ cases, with their pre-multiplied spectra nevertheless being significantly different. The spectra for the case $4 \mathrm{~L} / \mathrm{s}$ exhibit significant hills, associated with KHCSs, at all measurement locations, with their wavelengths and magnitudes increasing downstream (figure 17a, left-hand plot). This picture and hill wavelengths are fully consistent with the findings for KHCSs outlined in $\S 5.3$ and 5.4 (figures 14 and 15). In contrast, the case $16 \mathrm{~L} / \mathrm{s}$ is characterized by very weak signatures of KHCSs, which spectral signature becoming obvious only at $x^{*}=15.9$. This observation is also in full agreement with the findings reported in $\S 5.3$ and 5.4 (figures 14 and 15). Both cases $(4 \mathrm{~L} / \mathrm{s}$ and $16 \mathrm{~L} / \mathrm{s})$ do not show signs of VLSMs within FP, similar to $8 \mathrm{~L} / \mathrm{s}$ case.

\section{Momentum exchange and relaxation towards flow uniformity}

\subsection{Transverse exchange of streamwise momentum}

In the presence of transverse currents, the time- and depth-averaged transverse exchange of streamwise momentum $\tau_{\text {tot }}$ can be split into three components (e.g., Proust et al. 2013):

$$
\tau_{\text {tot }}=\overline{\frac{1}{D} \int_{0}^{D}-\rho u_{x} u_{y} d z}=-\rho\left(\overline{u_{x}^{\prime} u_{y}^{\prime}}\right)_{d}-\rho U_{x d} U_{y d}-\rho\left[U_{x}\left(U_{y}-U_{y d}\right)\right]_{d}
$$

where $u_{i}$ is the local instantaneous velocity component. Equation 7.1 is based on the decomposition of $u_{i}$ into three components: (1) time- and depth- averaged velocity $U_{i d}$, (2) deviation of the local time-averaged velocity from its depth-averaged counterpart, and (3) turbulent fluctuation $u_{i}^{\prime}$. Equation 7.1 explicitly shows that the total transverse exchange of momentum between $\mathrm{MC}$ and FP is driven by three key mechanisms: (1) transverse Reynolds stresses $\left(-\rho\left(\overline{u_{x}^{\prime} u_{y}^{\prime}}\right)_{d}\right) ;(2)$ transverse currents $\left(-\rho U_{x d} U_{y d}\right)$; and (3) $\operatorname{SCs}\left(-\rho\left[U_{x}\left(U_{y}-U_{y d}\right)\right]_{d}\right)$.

Figure 18 illustrates the relative contributions of these three key mechanisms at the interface. For all flow cases, shear layer (planform) turbulence and transverse currents have similar magnitudes in the depth-averaged momentum exchange, while the effect of SCs is negligible, consistent with the previous studies (e.g., van Prooijen et al. 2005; Dupuis et al. 2017a). For the depth-uniform flow case $8 \mathrm{~L} / \mathrm{s}$, it should be noted that the contribution of $-\rho U_{x d} U_{y d}$ is non-zero in the first half of the flume, due to the shear layer development. With transverse currents towards FPs $(0 \mathrm{~L} / \mathrm{s}$ and $4 \mathrm{~L} / \mathrm{s})$, the momentum fluxes by the transverse Reynolds stresses and by the transverse currents have the same sign, both contributing to the FP flow acceleration and the hastened recovery to flow uniformity (figure 8). In contrast, with transverse currents towards MC $(12 \mathrm{~L} / \mathrm{s}, 16 \mathrm{~L} / \mathrm{s}$, and $20 \mathrm{~L} / \mathrm{s}$ ), the two mechanisms act against each other.

The transverse distributions of the three contributions to the depth-averaged transverse exchange of streamwise momentum in the MC are reported in figure 19a for the two extreme flow cases $(20 \mathrm{~L} / \mathrm{s}$ and $0 \mathrm{~L} / \mathrm{s})$ at $x^{*}=4.4$ and 15.9. At both $x$-positions, the contributions of shear layer (planform) turbulence and SCs are of the same order of magnitude. Focusing on the upstream part of the flow, the transverse currents provide the predominant contribution to the spanwise momentum exchange, irrespective of the flow 


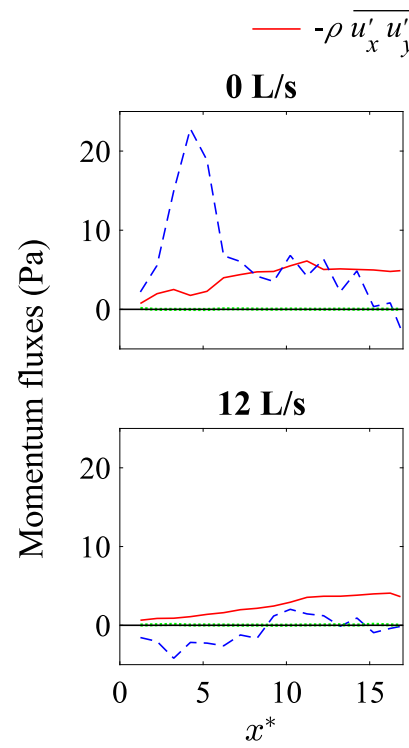

$---\rho U_{x d} U_{y d}$

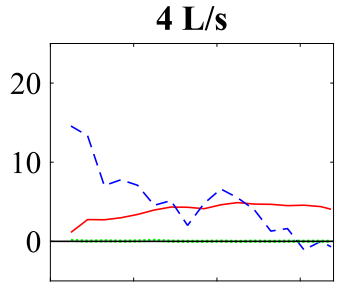

$16 \mathrm{~L} / \mathrm{s}$

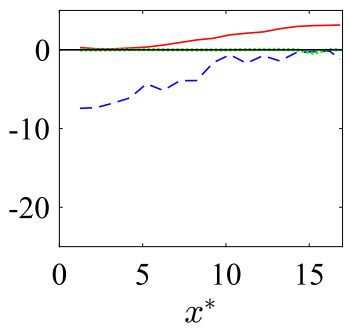

$-\rho\left[U_{x}\left(U_{y}-U_{y d}\right)\right]_{d}$

$8 \mathrm{~L} / \mathrm{s}$

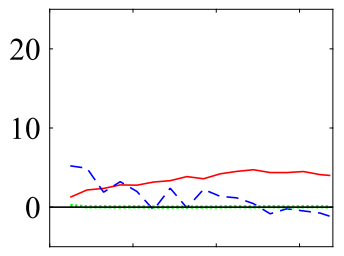

$20 \mathrm{~L} / \mathrm{s}$

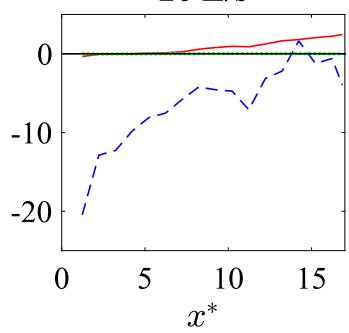

FIgURE 18. Contributions of shear layer (planform) turbulence $\left(-\rho\left(\overline{u_{x}^{\prime} u_{y}^{\prime}}\right)_{d}\right)$, transverse currents $\left(-\rho U_{x d} U_{y d}\right)$ and secondary currents $\left(-\rho\left[U_{x}\left(U_{y}-U_{y d}\right)\right]_{d}\right)$ to the total depth-averaged momentum exchange $\tau_{\text {tot }}$ (i.e., terms of Eq. 7.1). Measurements are taken at the interface.

direction (from $\mathrm{MC}$ to $\mathrm{FP}$ or vice versa). In addition, figure $19 \mathrm{a}$ shows that $-\rho U_{x d} U_{y d}$ and $-\rho\left(\overline{u_{x}^{\prime} u_{y}^{\prime}}\right)_{d}$ have the same sign for $0 \mathrm{~L} / \mathrm{s}$, both contributing to the deceleration of the MC flow, while these two terms are of opposite sign for $20 \mathrm{~L} / \mathrm{s}$.

Some examples of vertical distributions of the local momentum exchange terms by shear layer turbulence and SCs in the MC at $y^{*}=1.06$ are shown in figure $19 \mathrm{~b}$. These distributions highlight the significance of the SCs in the local transverse exchange of momentum which may well exceed the contribution of the Reynolds stresses. However, the vertical integrals of the SCs contributions are small compared to the action of transverse turbulence and can be negligible compared to the action of transverse currents, as highlighted above in figure 19a.

Last, figure 20 shows the effect of the lateral momentum exchange on the cross-sectional distribution of the time-averaged velocity $U_{x}$ in $\mathrm{MC}$ for $0 \mathrm{~L} / \mathrm{s}$ and $20 \mathrm{~L} / \mathrm{s}$ at $x^{*}=8.2$ (with normalization by the peak velocity). For $20 \mathrm{~L} / \mathrm{s}$, the deceleration of the MC flow is noticeable near the MC-FP interface in the upper flow region, owing to the very high values of the flux $-\rho U_{x d} U_{y d}$ upstream at $x^{*}=4.4$ (figure 19a). Slower water (mean flow) entering a faster flow in MC are responsible for this local decrease in velocity $U_{x}$. In contrast, for $0 \mathrm{~L} / \mathrm{s}$, as water is leaving the MC near the interface in the upper flow region, this decrease in $U_{x}$ is no more visible.

\subsection{Relaxation towards flow uniformity}

The plots in figures 18 and 19 have demonstrated that, in both MC and FP, the two key mechanisms of streamwise momentum exchanges act in the same direction for transverse currents directed towards FP, and act against each other for transverse currents directed towards MC. Indeed, with transverse currents towards the FP $(0 \mathrm{~L} / \mathrm{s}$ and $4 \mathrm{~L} / \mathrm{s})$, the transverse fluxes of streamwise momentum by the mean flow and by the KHCSs have the same sign over the FP (figure 18) and are two key mechanisms of momentum gain for the 
(a)
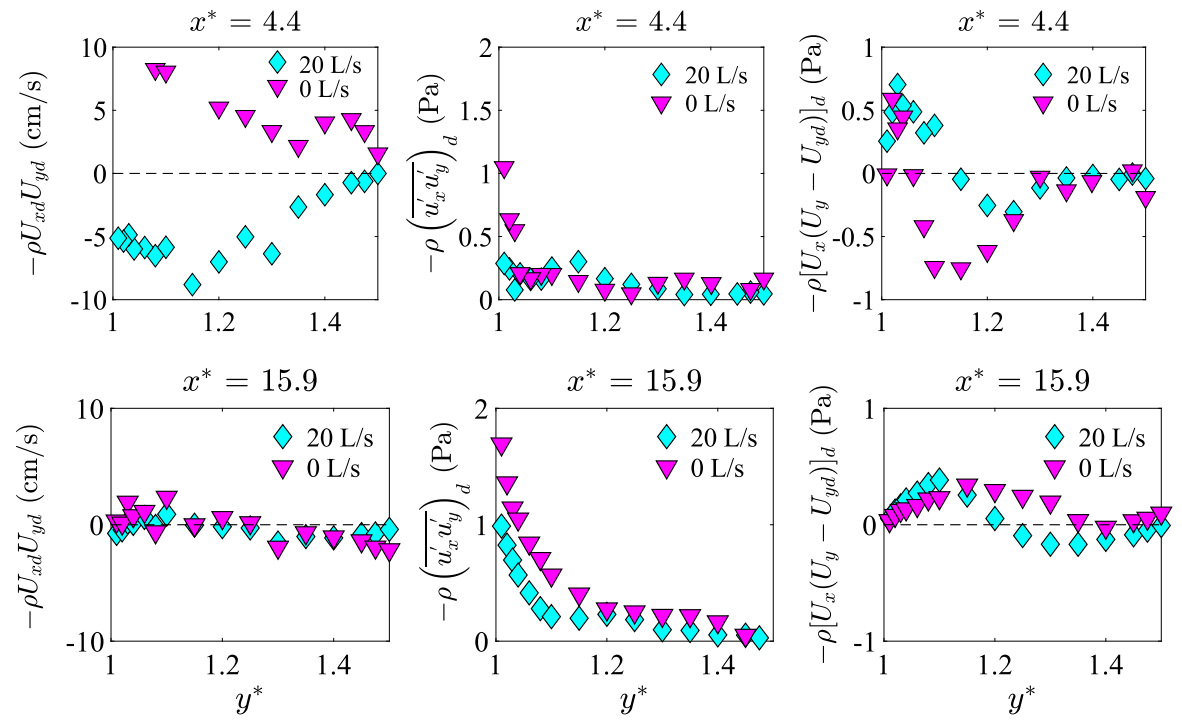

(b)
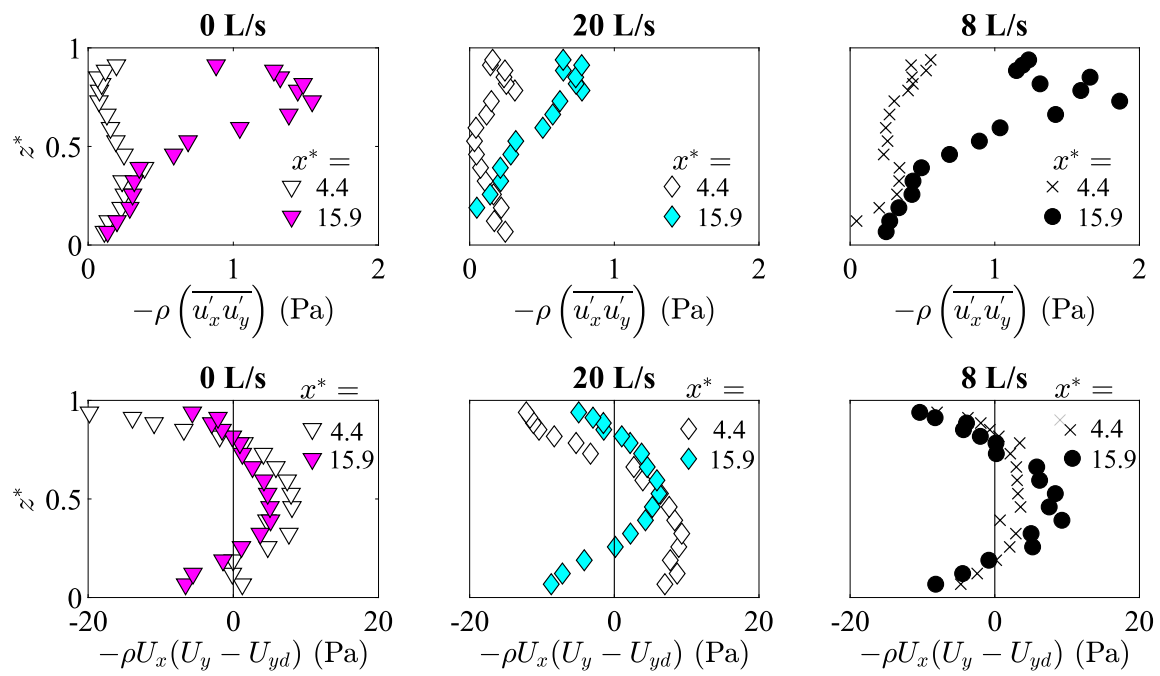

Figure 19. (a) Transverse distributions of depth-averaged momentum fluxes by the transverse currents, $-\rho U_{x d} U_{y d}$, by the shear layer turbulence, $-\rho\left(\overline{u_{x}^{\prime} u_{y}^{\prime}}\right)_{d}$, and by the SCs, $-\rho\left[U_{x}\left(U_{y}-U_{y d}\right)\right]_{d}$, for $20 \mathrm{~L} / \mathrm{s}(\diamond)$ and $0 \mathrm{~L} / \mathrm{s}(\nabla)$. (b) Vertical distributions of $-\rho \overline{u_{x}^{\prime} u_{y}^{\prime}}$ and $-\rho U_{x}\left(U_{y}-U_{y d}\right)$ at $y^{*}=1.06$ for 0,20 , and $8 \mathrm{~L} / \mathrm{s}$.

FP flow, while in the MC, these two fluxes always lead to the momentum reduction (figure 19). This results in a hastened development of the shear layer (figure 8). With transverse currents towards the MC $(12,16$, and $20 \mathrm{~L} / \mathrm{s})$, the KHCSs still serve as a mechanism of momentum supply to FP (figure 18), but the transverse flux of streamwise momentum by the mean flow represents momentum (and mass) loss. In MC, the transverse fluxes of streamwise momentum by transverse currents and KHCSs are also of opposite signs. As the two mechanisms of momentum transfer act in the opposite directions in both sub-sections, the development of the shear layer is delayed (figure 8).

The asymmetry in the relaxation towards flow uniformity (depending on the transverse 
(a)

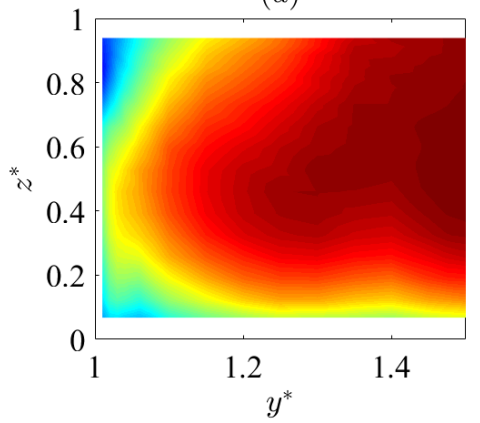

(b)

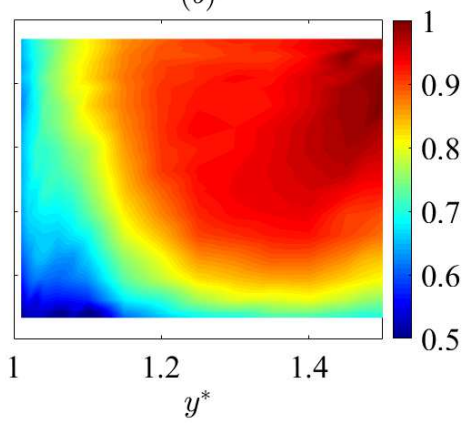

FiguRE 20. Cross-sectional distributions of the time-averaged streamwise velocity, $U_{x}$, normalized by the peak velocity, $U_{x, \max }$, across a half-MC at $x^{*}=8.2$. (a) $20 \mathrm{~L} / \mathrm{s}$; (b) $0 \mathrm{~L} / \mathrm{s}$.
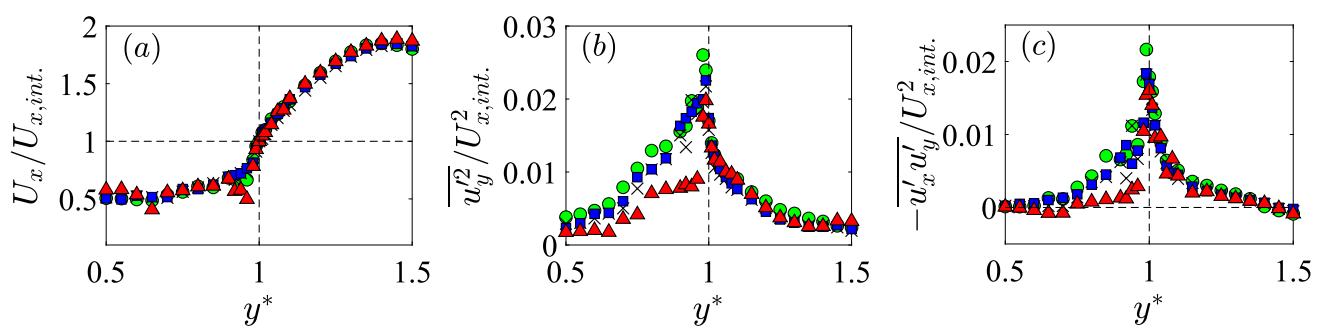

Figure 21. Transverse profiles of dimensionless (a) mean streamwise velocity, $U_{x} / U_{x, \text { int }}$, (b) transverse turbulence intensity, $\overline{u_{y}^{\prime 2}} / U_{x, i n t}^{2}$, and (c) transverse Reynolds shear stress, $-\left(\overline{u_{x}^{\prime} u_{y}^{\prime}}\right) / U_{x, \text { int }}^{2}$, at $x^{*}=16.4$ and $z^{*}=0.91$ for cases $4 \mathrm{~L} / \mathrm{s}(\circ), 8 \mathrm{~L} / \mathrm{s}(\times), 12 \mathrm{~L} / \mathrm{s}(\square)$, and 16 $\mathrm{L} / \mathrm{s}(\triangle)$. The standard errors in $U_{x}, \overline{u_{y}^{\prime 2}}$, and $-\left(\overline{u_{x}^{\prime} u_{y}^{\prime}}\right)$, are around $1 \%, 4 \%$, and $10 \%$.
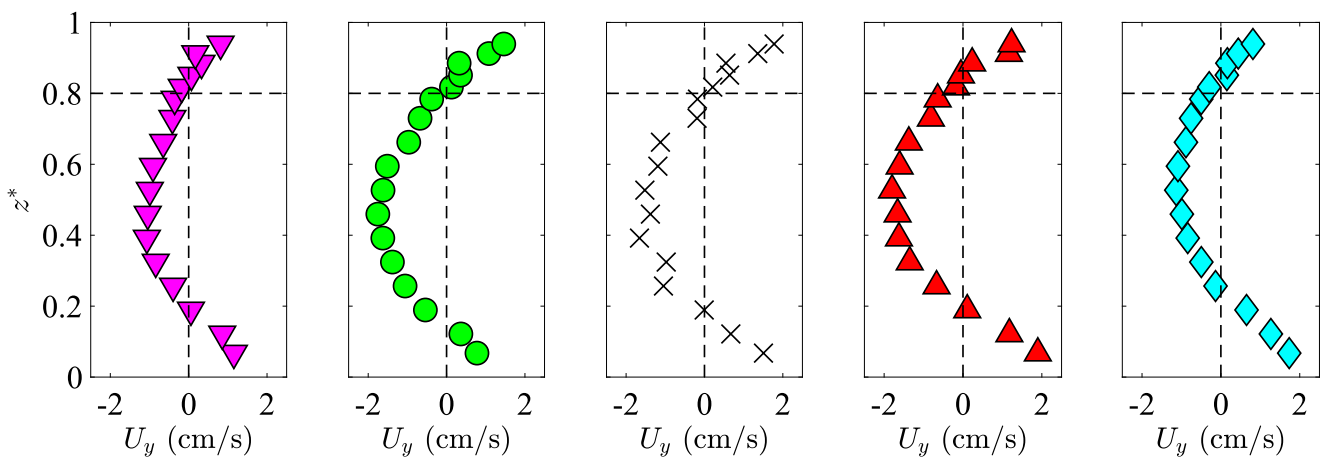

Figure 22. Vertical distributions of the time-averaged transverse velocity, $U_{y}$, at $y^{*}=1.06$ (in $\mathrm{MC})$ and $x^{*}=15.9$, for cases: $0 \mathrm{~L} / \mathrm{s}(\nabla) ; 4 \mathrm{~L} / \mathrm{s}(\circ) ; 8 \mathrm{~L} / \mathrm{s}(\times) ; 16 \mathrm{~L} / \mathrm{s}(\triangle)$; and $20 \mathrm{~L} / \mathrm{s}(\diamond)$. The standard sampling error in $U_{y}$ is around $9 \%$.

currents direction) is also noticeable when focusing on the turbulence statistics. For instance, at the MC/FP interface (figure 5), the depth-averaged transverse turbulence intensity and transverse Reynolds stress for $0 \mathrm{~L} / \mathrm{s}$ are closer to the uniform flow values $(8 \mathrm{~L} / \mathrm{s})$ than the corresponding values for $16 \mathrm{~L} / \mathrm{s}$. The same trends hold when comparing $4 \mathrm{~L} / \mathrm{s}$ to $12 \mathrm{~L} / \mathrm{s}$, with $8 \mathrm{~L} / \mathrm{s}$ as a reference flow.

The transverse profiles of the flow parameters in the last measuring section at $z^{*}=0.91$ provide additional insights (figure 21 ), when putting aside the two extreme cases. The 
mean velocity profiles fairly collapse while significant discrepancies can be observed for turbulence statistics between $16 \mathrm{~L} / \mathrm{s}$ and the three other cases, particularly over the FP. For $16 \mathrm{~L} / \mathrm{s}$, the low values of the Reynolds shear stress and transverse turbulence intensity highlight the spatial delay in the development of the KHCSs along the flow (figure 15). Mean velocity profiles are thus far less sensitive to the upstream flow history than the turbulence statistics profiles. As a result, the Boussinesq assumption and the related eddy viscosity concept, which have been widely used under uniform flow conditions (e.g., Knight \& Shiono 1990; Shiono \& Knight 1991; van Prooijen et al. 2005; Fernandes et al. 2014), will not be valid in the presence of noticeable transverse currents.

Finally, the asymmetry in the relaxation can also be observed in the SCs patterns, since over the FP at mid length of the flume $\left(x^{*}=8.4\right)$, the longitudinal FP vortex is fully developed for $4 \mathrm{~L} / \mathrm{s}$ but is at its initial stage of development for case $20 \mathrm{~L} / \mathrm{s}$ (compare figures $9 \mathrm{~b}, 10 \mathrm{~b}$, and $11 \mathrm{~b}$ ). The same result holds in MC, as at the same position in MC $\left(x^{*}=8.4\right)$, three SCs cells are observed for 0,4 , and $8 \mathrm{~L} / \mathrm{s}$, but only one cell for 16 and 20 $\mathrm{L} / \mathrm{s}$. On the other hand, the equilibrium is achieved in $\mathrm{MC}$ for all initially non-uniform flows at $x^{*}=15.9$, as shown in figure 22 . The $U_{y}$-distributions for the non-uniform cases at $y^{*}=1.06$, i.e., at a location where a peak $U_{y}$-value is observed across the MC for the uniform case $8 \mathrm{~L} / \mathrm{s}$ (figure 10a), are very similar to that of the uniform case.

Overall, the asymmetry in the relaxation towards uniformity depending on the transverse currents direction was observed for: flow depth, shear layer widths, turbulence statistics, SCs patterns, and KHCSs. For the latter, transverse currents are not directly responsible for the delay in the KHCSs development. This is due to the low values of dimensionless velocity shear $(\lambda<0.3)$ in the upstream part of the flume for the flows with a large excess in FP flow $(16$ and $20 \mathrm{~L} / \mathrm{s}$ ), which prevents the emergence of KHCSs.

\section{Conclusions}

Compound open-channel flows have been investigated in a large laboratory flume. The experiments covered three representative scenarios (figure 23): uniform flow conditions, i.e., with unchanging flow depth in the streamwise direction; non-uniform flow with transverse current from floodplain (FP) to main channel (MC); and non-uniform flow with transverse current from MC to FP. The transverse currents are induced by unbalanced inflow conditions. The main attention of the study was on clarifying four questions: 1) what is the effect of transverse currents on the planform shear layer between MC and FP and the horizontal Kelvin-Helmholtz type coherent structures (KHCSs)? 2) what are the conditions for the emergence and development of KHCSs within the shear layer in the presence of flow non-uniformity? 3) what is the effect of the transverse flow on the SC cells and how this effect depends on the magnitude and direction of the transverse currents? 4) does the turbulence structure outside the shear layer exhibit the presence of large (LSMs) or very large scale motions (VLSMs)?

First, it is found that transverse currents can entirely displace the shear layer over the FP (figure 23c) or in the MC (figure 23b), as they are the primary contribution to transverse momentum exchange in the upstream half flume. However, they do not alter in the same way the KHCSs development. This is reflected in a mismatch between shear layer extent and KHCSs length scales in both MC and FP. For instance, at significant currents towards the MC (figures $14 \mathrm{e}, 14 \mathrm{j}$, and $23 \mathrm{~b}$ ) it was shown that in the upstream part of the flow, the transverse length scale of the KHCSs in MC, $\delta_{m}^{C S}$, is zero, while the mixing layer width in $\mathrm{MC}, \delta_{m}$, is well noticeable due to the strong momentum exchange by the mean flow; in the downstream part of the flow, the mixing layer width over the $\mathrm{FP}, \delta_{f}$, can be close to zero while KHCSs extend largely over the FP. 
(a)

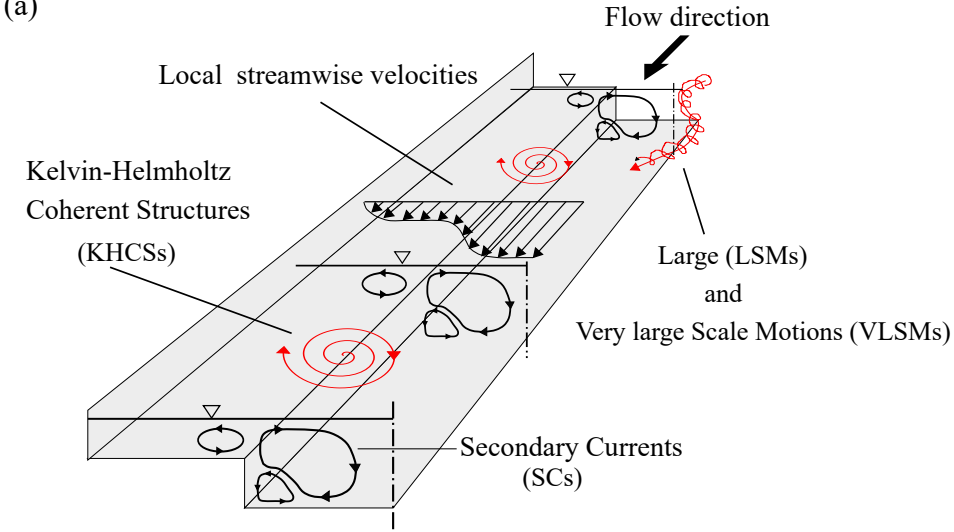

(b)

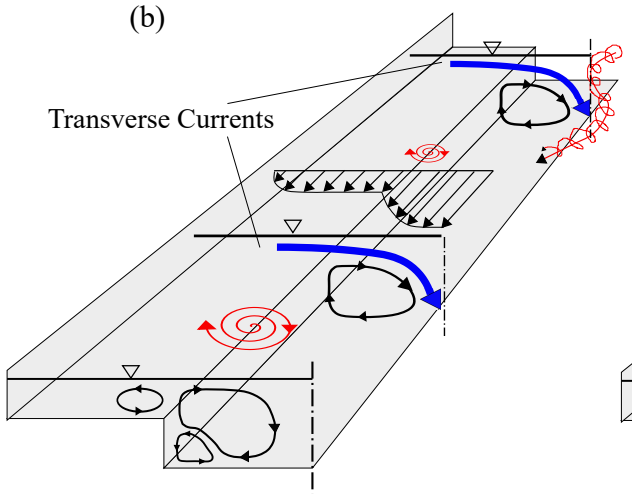

(c)

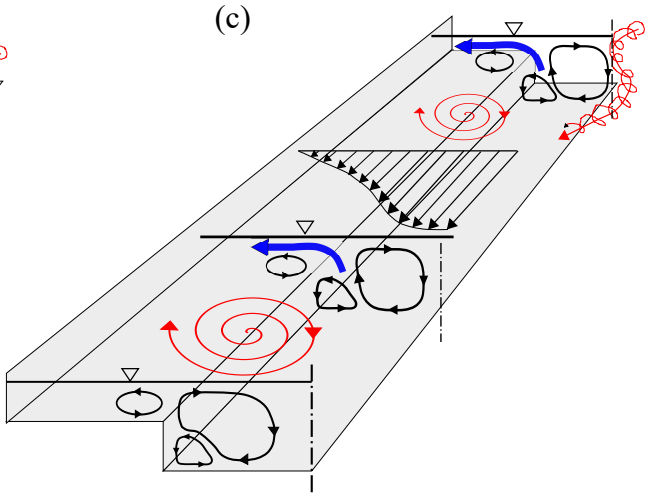

FiguRE 23. Key mechanisms responsible for the flow structure in depth-uniform reference flow $(a)$ and non-uniform flows with transverse currents towards MC $(b)$ and towards $\mathrm{FP}(c)$.

Second, for both uniform and non-uniform flows, the emergence of KHCSs is essentially driven by the velocity shear between the two 'ambient' streams outside the shear layer, as quantified with dimensionless shear parameter $\lambda=\left(U_{x 2}-U_{x 1}\right) /\left(U_{x 2}+U_{x 1}\right)$. The KHCSs emerge once $\lambda$ exceeds 0.3 above which KHCSs length-scales increase with the velocity shear. Unlike mixing layers behind vertical splitter plates in rectangular cross-section channels, flow confinement in a compound channel has no effect on KHCSs.

Third, three well-established SC cells are observed in uniform flow: one longitudinal helical motion over the FP near the interface; and two cells in MC, a small cell near the bottom at the $\mathrm{MC}$ corner, and a larger cell extending over the whole depth (figure 23a). These cells are both induced by flow heterogeneity and planform shear layer turbulence anisotropy. With transverse currents towards FP (figure 23c), the three cells are unaltered, in keeping with the high level of shear layer turbulence (related to high velocity shear values). With transverse currents towards MC (figure 23b), the three cells are replaced by a single large cell in MC, induced by the horizontal shearing between the upper mean flow and the lower mean flow below the bank full stage in MC. The integral contribution of SCs to the momentum exchange at the MC/FP interface is zero but can be of the same order of magnitude as the Reynolds shear stresses in MC for the three scenarios.

Fourth, overall, the data for the uniform flow case suggest that in the initial development of KHCSs, SCs, LSMs and VLSMs in MC (figure 23a), the latter two appear to be fairly competitive. The spectral signatures of LSMs and VLSMs are detectable at the 
upstream section of the flume within the central part of MC but they quickly disappear along the flow being suppressed by simultaneous development of KHCSs and SCs. At the same time, within FP the effects of KHCSs became already dominant in the upstream flow region suppressing conditions allowing VLSMs to form. The suppressing influence of KHCSs over FP is likely supplemented by the artificial grass effect that could help to dump VLSMs. Thus, the data suggest that the transverse shear layer, KHCSs and SCs become the most dominant features of the flow dynamics within a fairly short distance from the flume entrance. The effect of the transverse currents on LSMs and VLSMs and their downstream evolution in MC is very weak (if any). As for FP, similar to the uniform flow case, no signatures of VLSMs are noted for all non-uniform cases.

Last, the direction of the transverse currents and their magnitude appeared to be of the critical importance for relaxation of initially non-uniform flows to equilibrium conditions. The equilibrium in relation to flow depth, shear layer, turbulence statistics and SCs is reaching over a shorter distance with transverse currents towards the FP than with currents towards the MC. This asymmetry in the relaxation is due to the fact that, in both MC and FP, KHCSs and transverse currents act in the same direction for transverse currents directed towards FP (both accelerate the FP flow and decelerate the MC flow) while they act against each other with transverse currents directed towards MC. Figure 23 provides overall conceptual presentation of the key momentum transfer mechanisms observed in the studied flow scenarios.

The authors wish to thank Fabien Thollet and Alexis Buffet for their technical support, and the anonymous referees for their valuable comments that have helped to improve the paper. This research work was funded by the French National Research Agency under grant NANR-14-CE03-0010 (Flowres ANR project, https://flowres.irstea.fr/en/).

Declaration of Interests. The authors report no conflict of interest.

\section{REFERENCES}

ACKers, P. 1993 Flow formulae for straight two-stage channels. J. Hydraul. Res. 31 (4), 504531.

Adrian, R. J. \& MARusic, I. 2012 Coherent structures in flow over hydraulic engineering surfaces. J. Hydraul. Res. 50 (5), 451-464.

Akutina, Y., Eiff, O., Moulin, F. \& Rouzes, M. 2019 Lateral bed-roughness variation in shallow open-channel flow with very low submergence. Environ. Fluid Mech. https://doi.org/10.1007/s10652-019-09678-w.

Azevedo, Ricardo, Roja-Solórzano, Luís R. \& Bento Leal, João 2017 Turbulent structures, integral length scale and turbulent kinetic energy (tke) dissipation rate in compound channel flow. Flow Meas. Instrum. 57 (Supplement C), 10-19.

BAtChelor, G.K. 1969 Computation of the energy spectrum in homogeneous two-dimensional turbulence. Phys. Fluids 12 (Suppl. II), 233-239.

Besio, G., Stocchino, A., Angiolani, S. \& Brocchini, M. 2012 Transversal and longitudinal mixing in compound channels. Water Resour. Res. 48 (W12517), 15 pp.

Bousmar, D. 2002 Flow modelling in compound channels / momentum transfer between main channel and prismatic or non-prismatic floodplains. Ph-d thesis, université catholique de louvain, faculté des sciences appliquées.

Bousmar, D., Riviere, N., Proust, S., Paquier, A., Morel, R. \& Zech, Y. 2005 Upstream discharge distribution in compound-channel flumes. J. Hydraul. Eng., ASCE 131 (5), 408-412.

Bousmar, D., Wilkin, N., Jacquemart, J.H. \& Zech, Y. 2004 Overbank flow in symmetricaly narrowing floodplains. J. Hydraul. Eng. 130 (4), 305-312.

Brown, G.L. \& Roshko, A. 1974 On density effects and large structure in turbulent mixing layers. J. Fluid Mech. 64 (part 4), 775-816. 
Cameron, S., Nikora, V. \& Marusic, I. 2019 Drag forces on a bed particle in open-channel flow: Effects of pressure spatial fluctuations and very large scale motions. J. Fluid Mech. 863, 494-512.

Cameron, S.M., Nikora, V.I. \& Stewart, M.T. 2017 Very-large-scale motions in rough-bed open-channel flow. J. Fluid Mech. 814, 416-429.

Caroppi, G., Västilë, K., Järvelë, J., Rowinski, P.M. \& Giugni, M. 2019 Turbulence at water-vegetation interface in open channel flow: Experiments with natural-like plants. Adv. Water Resour. 127 (May 2019), 180-191.

Champagne, F.H., PaO, Y.H. \& Wygnanski, I.J. 1976 On the two-dimensional mixing region. J. Fluid Mech. $\mathbf{7 4}$ (part 2), 209-250.

Chu, V.H. \& BABARutsi, S. 1988 Confinement and bed-friction effects in shallow turbulent mixing layers. J. Hydraul. Eng. 114 (10), 1257-1274.

Constantinescu, G., Miyawaki, S., Rhoads, B., Sukhodolov, A. \& Kirkil, G. 2011 Structure of turbulent flow at a river confluence with momentum and velocity ratios close to 1: Insight provided by an eddy-resolving numerical simulation. Water Resour. Res. 47 (W05507), 1-16.

Das, B. S., Devi, K. \& Khatua, K. K. 2019 Prediction of discharge in converging and diverging compound channel by gene expression programming. ISH J. Hydraul. Eng. pp. 1-11.

Dracos, T., Giger, M. \& Jirka, G.H. 1992 Plane turbulent jets in a bounded fluid layer. J. Fluid Mech. 241, 587-614.

Dupuis, V., Proust, S., Berni, C. \& Paquier, A. 2016 Combined effects of bed friction and emergent cylinder drag in open channel flow. Environ. Fluid Mech. 16 (6), 1173-1193.

Dupuis, V., Proust, S., Berni, C. \& Paquier, A. 2017a Mixing layer development in compound channel flows with submerged and emergent rigid vegetation over the floodplains. Exp. Fluids 58 (30), 1-18.

Dupuis, V., Proust, S., Berni, C. \& Paquier, A. 2017b Compound channel flow with a longitudinal transition in hydraulic roughness over the floodplains. Environ. Fluid Mech. 17, 903-928.

Elliot, S.C.A. \& Sellin, R. H. J. 1990 Serc flood channel facility : skewed flow experiments. J. Hydraul. Res. 28 (2), 197-214.

Fernandes, J. N., Leal, J. B. \& Cardoso, A.H. 2014 Improvement of the lateral distribution method based on the mixing layer theory. Adv. Water Resour. 69, 159-167.

Goring, Derek G \& Nikora, Vladimir I 2002 Despiking acoustic doppler velocimeter data. J. Hydraul. Eng. 128 (1), 117-126.

Hutchins, N. \& MARusic, I. 2007 Evidence of very long meandering features in the logarithmic region of turbulent boundary layers. J. Fluid Mech. 579, 1-28.

IkedA, S. \& MCEwan, I.K. 2009 Flow and sediment transport in Compound Channels. IAHR International Association of hydraulic Engineering and Research, CRC Press, Madrid, Spain.

Kim, K.C. \& Adrian, R.J. 1999 Very large-scale motion in the outer layer. Phys. Fluids 11 (2), 417-422.

Knight, D.W. \& Demetriou, J.D. 1983 Floodplain and main channel flow interaction. J. Hydraul. Eng., ASCE 109 (8), 1073-1092.

Knight, D.W. \& Shiono, K. 1990 Turbulence measurements in a shear layer region of a compound channel. J. Hydraul. Res. 28 (2), 175-194.

Kraichnan, R. 1967 Inertial ranges in two-dimensional turbulence. Phys. Fluids 10, 1417-1428.

LESIEUR, M. 2013 Turbulence. EDP sciences.

McDonough, J.M. 2007 Introductory lectures on turbulence. Physics, Mathematics and Modeling. University of Kentucky: Departments of Mechanical Engineering and Mathematics.

MentA, R.D. 1991 Effect of velocity ratio on plane mixing layer development: Influence of the splitter plate wake. Exp. Fluids 10, 194-204.

Nezu, I., Onitsuka, K. \& Iketani, K. 1999 Coherent horizontal vortices in compound open channel flows. In Hydraulic modeling (ed. I. W. Seo V. P. Singh \& J. H. Sonu), pp. 17-32. Water Resources Publications, Colorado, USA.

Nicollet, G. \& UAN, M. 1979 Ecoulements permanents à surface libre en lit composés. La Houille Blanche (1), 21-30. 
O’Neill, P.L., Nicolaides, D., Honnery, D. \& Soria, J. 2004 Autocorrelation functions and the determination of integral length with reference to experimental and numerical data. In 15th Australasian Fluid Mechanics Conference, p. 4.

Oster, D. \& Wygnanski, I. 1982 The forced mixing layer between parallel streams. J. Fluid Mech. 123, 91-130.

Peltier, Y., Proust, S., Rivière, N., Paquier, A. \& Shiono, K. 2013a Turbulent flows in straight compound open-channel with a transverse embankment on the floodplain. $J$. Hydraul. Res. 51 (4), 446-458.

Peltier, Y., Rivière, N., Proust, S., Mignot, E., Paquier, A. \& Shiono, K. 2013b Estimation of the error on the mean velocity and on the reynolds stress due to a misoriented adv probe in the horizontal plane: case of experiments in a compound openchannel. Flow Meas. Instrum. 34, 34-41.

van Prooijen, B.C., Battjes, J.A. \& Uijttewaal, W.S.J. 2005 Momentum exchange in straight uniform compound channel flow. J. Hydraul. Eng. 131 (3), 175-183.

Proust, S. 2005 Ecoulements non-uniformes en lits composés : effets de variations de largeur du lit majeur. PhD thesis, INSA de Lyon.

Proust, S., Bousmar, D., Riviere, N., Paquier, A. \& Zech, Y. 2010 Energy losses in compound open channels. Adv. Water Resour. 33, 1-16.

Proust, S., Fernandes, J. N., Leal, J. B., Rivière, N. \& Peltier, Y. 2017 Mixing layer and coherent structures in compound channel flows: effects of transverse flow, velocity ratio and vertical confinement. Water Resour. Res. 53 (4), 3387-3406.

Proust, S., Fernandes, J. N., Peltier, Y., Leal, J. B., Rivière, N. \& Cardoso, A.H. 2013 Turbulent non-uniform flows in straight compound open-channels. J. Hydraul. Res. 51 (6), 656-667.

Proust, S., Riviere, N., Bousmar, D., Paquier, A., Zech, Y. \& Morel, R. 2006 Flow in compound channel with abrupt floodplain contraction. J. Hydraul. Eng. 132 (9), 958-970.

SELlin, R.H.J. 1964 A laboratory investigation into the interaction between the flow in the channel of a river and that over its flood plain. La Houille Blanche (7), 793-802.

Shiono, K. \& Knight, D.W. 1991 Turbulent open channel flows with variable depth across the channel. J. Fluid Mech. 222, 617-646.

Shiono, K. \& Muto, Y. 1998 Complex flow mechanisms in compound meandering channels with overbank flow. J. Fluid Mech. 376, 221-226.

Soldini, L., Piattella, A., Mancinelli, A., Bernetti, R. \& Brocchini, M. 2004 Macrovortices-induced horizontal mixing in compound channels. Ocean Dyn. 54 (3), 333339.

Stocchino, A., Besio, G., Angiolani, S. \& Brocchini, M. 2011 Lagrangian mixing in straight compound channels. J. Fluid Mech. 675, 168-198.

Stocchino, A. \& Brocchini, M. 2010 Horizontal mixing of quasi-uniform straight compound channel flows. J. Fluid Mech. 643, 425-435.

Sturm, T.W. \& SAdiQ, A. 1996 Water surface profiles in compound channels with multiple critical depths. J. Hydraul. Eng., ASCE 122 (12), 703-709.

Tabeling, P. 2002 Two-dimensional turbulence: a physicist approach. Phys. Rep. 362, 1-62.

Tominaga, A. \& Nezu, I. 1991 Turbulent structure in compound open-channel flows. J. Hydraul. Eng. 117 (1), 21-41.

Truong, S. H., Uijttewaal, W. S. J. \& Stive, M. J. F. 2019 Exchange processes induced by large horizontal coherent structures in floodplain vegetated channels. Water Resour. Res. 55 (3), 2014-2032.

UijtTewaAL, W.S.J. \& BooiJ, R. 2000 Effects of shallowness on the development of free-surface mixing layers. Phys. Fluids 12 (2), 392-420.

ZAMPIRON, A. 2019 Hydraulic resistance and flow structure in open-channel flow over streamwise ridges. $\mathrm{PhD}$ thesis, University of Aberdeen.

Zampiron, A., Cameron, S. \& V., Nikora 2019 Secondary currents and very-large-scale motions in open-channel flow over streamwise ridges. J. Fluid Mech. (Resubmitted).

Zheleznyakov, G.V. 1965 Relative deficit of mean velocity of unstable river flow, kinematic effect in river beds with flood plain. In Proc. 11th IAHR Congress, Leningrad. 